FREI M.M.

\title{
WICK CALCULUS ON SPACES OF REGULAR GENERALIZED FUNCTIONS OF LÉVY WHITE NOISE ANALYSIS
}

\begin{abstract}
Many objects of the Gaussian white noise analysis (spaces of test and generalized functions, stochastic integrals and derivatives, etc.) can be constructed and studied in terms of so-called chaotic decompositions, based on a chaotic representation property (CRP): roughly speaking, any square integrable with respect to the Gaussian measure random variable can be decomposed in a series of Itô's stochastic integrals from nonrandom functions. In the Lévy analysis there is no the CRP (except the Gaussian and Poissonian particular cases). Nevertheless, there are different generalizations of this property. Using these generalizations, one can construct different spaces of test and generalized functions. And in any case it is necessary to introduce a natural product on spaces of generalized functions, and to study related topics. This product is called a Wick product, as in the Gaussian analysis.

The construction of the Wick product in the Lévy analysis depends, in particular, on the selected generalization of the CRP. In this paper we deal with Lytvynov's generalization of the CRP and with the corresponding spaces of regular generalized functions. The goal of the paper is to introduce and to study the Wick product on these spaces, and to consider some related topics (Wick versions of holomorphic functions, interconnection of the Wick calculus with operators of stochastic differentiation). Main results of the paper consist in study of properties of the Wick product and of the Wick versions of holomorphic functions. In particular, we proved that an operator of stochastic differentiation is a differentiation (satisfies the Leibniz rule) with respect to the Wick multiplication.

Key words and phrases: Lévy process, stochastic differentiation, Wick product.
\end{abstract}

Vasyl Stefanyk Precarpathian National University, 57 Shevchenka str., 76018, Ivano-Frankivsk, Ukraine

E-mail: mashadyriv@ukr.net

\section{INTRODUCTION}

Due to development of physics and mathematics (in particular, of the quantum field theory, of the mathematical physics, of the theory of random processes) there is a need to develop a theory of test and generalized functions of infinitely many variables. There are different approaches to building of such a theory. Correspondingly, different spaces of test and generalized functions are the object of study. One of the most successful approaches consists in building of the just now mentioned spaces in such a way that the natural pairing between test and generalized functions is generated by integration with respect to some probability measure on a dual nuclear space (in particular, on a dual Schwartz space). First it was the standard Gaussian measure (the measure of a Gaussian white noise), the corresponding theory is called the Gaussian white noise analysis (see, e.g., $[10,21]$ ); then it were realized numerous generalizations. In particular, important for applications results can be obtained if as the above-mentioned 
measure one uses a so-called Lévy white noise measure (e.g., $[4,5])$, the corresponding theory is called the Léry white noise analysis.

An important role in the Gaussian analysis belongs to a so-called chaotic representation property (CRP): roughly speaking, any square integrable with respect to the Gaussian measure random variable can be decomposed in a series of Itô's stochastic integrals from nonrandom functions. In particular, the CRP can be used in order to construct the extended Skorohod stochastic integral $[13,27]$ and the Hida stochastic derivative [10].

Unfortunately, in the Lévy analysis there is no the CRP [29] (except Gaussian and Poissonian particular cases). Nevertheless, there are different approaches to a generalization of this property: Itô's approach [12], Nualart-Schoutens' approach [24,25], Lytvynov's approach [23], Oksendal's approach $[4,5]$, etc. The interconnections between these generalizations of the CRP are described in, in particular, $[1,4,5,17,23,28,30]$.

One can use different generalizations of the CRP and construct different spaces of test and generalized functions in the Lévy analysis, depending on the purpose of the research. And in any case, for solving of some problems, or even simply for the completeness of the theory, it is necessary to introduce a natural product on spaces of generalized functions, and to study related topics. In the classical Gaussian analysis such a product, known as a Wick product, can be introduced with use of symmetric tensor products of kernels from natural decompositions of generalized functions (e.g., [22]). But in a general Lévy analysis the situation is more complicated: now the construction of a product on spaces of generalized functions appreciably depends on the construction of the just now mentioned spaces that, by-turn, depends, in particular, on the selected generalization of the CRP. For example, elements of the Lévy analysis in terms of Oksendal's generalization of the CRP and, in particular, the corresponding Wick product and related topics, are considered in [4,5].

In this paper we deal with so-called regular parametrized Kondratiev-type spaces of generalized functions of the Lévy white noise analysis [16], which are constructed with use of Lytvynov's generalization of the CRP. The goal of the paper is to introduce and to study a natural product (a Wick product) on these spaces, and to consider some related topics (Wick versions of holomorphic functions, stochastic equations with Wick type nonlinearities, interconnection of the Wick calculus with operators of stochastic differentiation). Main results of the paper consist in study of properties of the Wick product and of the Wick versions of holomorphic functions. In particular, we proved that an operator of stochastic differentiation is a differentiation (satisfies the Leibniz rule) with respect to the Wick multiplication.

Note that, as distinguished from the Gaussian case, now the symmetric tensor product of kernels from natural decompositions of generalized functions is indeterminated, therefore we introduce an applicable generalization of this product, by analogy with a so-called Gamma white noise analysis [15] and a more general Meixner white noise analysis [14].

The paper is organized in the following manner. In the first section we recall necessary notions, definitions and statements. Namely, we introduce a Lévy process $L$ and convenient for our considerations probability space connected with $L$; describe in detail Lytvynov's generalization of the CRP; consider a regular parametrized rigging of $\left(L^{2}\right)$, and the stochastic integrals, derivatives, and operators of stochastic differentiation on the spaces that belong to this rigging. The second section is devoted to the Wick calculus: in the first subsection we introduce and study the Wick product and the Wick versions of holomorphic functions on the spaces of regular generalized functions; in the second subsection we study an interconnection 
between the Wick calculus and the operator of stochastic differentiation.

\section{PRELIMINARIES}

In this paper we accept on default that signs $\|\cdot\|_{H}$ or $|\cdot|_{H}$ denote the norm in a space $H$; a sign $(\cdot, \cdot)_{H}$ denotes the scalar product in $H$; signs $\langle\cdot, \cdot\rangle_{H}$ or $\langle\langle\cdot, \cdot\rangle\rangle_{H}$ denote the dual pairing generated by the scalar product in $H$.

\subsection{Lévy processes}

Set $\mathbb{R}_{+}:=[0,+\infty)$. Consider a real-valued locally square integrable Lévy process $L=$ $\left(L_{t}\right)_{t \in \mathbb{R}_{+}}$(i.e. a random process on $\mathbb{R}_{+}$with stationary independent increments and such that $L_{0}=0$ ) without Gaussian part and drift. As is known (e.g., [5]), the characteristic function of $L$ is

$$
\mathbb{E}\left[e^{i \theta L_{t}}\right]=\exp \left[t \int_{\mathbb{R}}\left(e^{i \theta x}-1-i \theta x\right) v(d x)\right] .
$$

Here $v$ is the Lévy measure of $L$, which is a measure on $(\mathbb{R}, \mathcal{B}(\mathbb{R}))$, here and below $\mathcal{B}$ denotes the Borel $\sigma$-algebra; $\mathbb{E}$ denotes the expectation. We assume that $v$ is a Radon measure whose support contains an infinite number of points, $v(\{0\})=0$, there exists $\varepsilon>0$ such that

$$
\int_{\mathbb{R}} x^{2} e^{\varepsilon|x|} v(d x)<\infty
$$

and

$$
\int_{\mathbb{R}} x^{2} v(d x)=1
$$

Let us define the measure of the white noise of $L$. By $\mathcal{D}$ denote the set of all real-valued infinite-differentiable functions on $\mathbb{R}_{+}$with compact supports. As is known, $\mathcal{D}$ can be endowed by the projective limit topology generated by a family of Sobolev spaces (e.g., [3]). Let $\mathcal{D}^{\prime}$ be the set of linear continuous functionals on $\mathcal{D}$. For $\omega \in \mathcal{D}^{\prime}$ and $\varphi \in \mathcal{D}$ denote $\omega(\varphi)$ by $\langle\omega, \varphi\rangle$; note that one can understand $\langle\cdot, \cdot\rangle$ as the dual pairing generated by the scalar product in the space $L^{2}\left(\mathbb{R}_{+}\right)$of (classes of) square integrable with respect to the Lebesgue measure real-valued functions on $\mathbb{R}_{+}$(e.g., [3]). The notation $\langle\cdot, \cdot\rangle$ will be preserved for dual pairings in tensor powers of riggings of $L^{2}\left(\mathbb{R}_{+}\right)$and in tensor powers of complexifications of such riggings.

Definition 1. A probability measure $\mu$ on $\left(\mathcal{D}^{\prime}, \mathcal{C}\left(\mathcal{D}^{\prime}\right)\right)$, where $\mathcal{C}$ denotes the cylindrical $\sigma$ algebra, with the Fourier transform

$$
\int_{\mathcal{D}^{\prime}} e^{i\langle\omega, \varphi\rangle} \mu(d \omega)=\exp \left[\int_{\mathbb{R}_{+} \times \mathbb{R}}\left(e^{i \varphi(u) x}-1-i \varphi(u) x\right) d u v(d x)\right], \quad \varphi \in \mathcal{D},
$$

is called the measure of a Léry white noise.

The existence of $\mu$ follows from the Bochner-Minlos theorem (e.g., [11]), this proved in [23]. Below we assume that the $\sigma$-algebra $\mathcal{C}\left(\mathcal{D}^{\prime}\right)$ is completed with respect to $\mu$, i.e. we take the completion of $\mathcal{C}\left(\mathcal{D}^{\prime}\right)$ and preserve for this completion the previous designation. So, now $\mathcal{C}\left(\mathcal{D}^{\prime}\right)$ contains all subsets of all measurable sets $O$ such that $\mu(O)=0$.

Denote by $\left(L^{2}\right):=L^{2}\left(\mathcal{D}^{\prime}, \mathcal{C}\left(\mathcal{D}^{\prime}\right), \mu\right)$ the space of (classes of) complex-valued square integrable with respect to $\mu$ functions on $\mathcal{D}^{\prime}$. Let $f \in L^{2}\left(\mathbb{R}_{+}\right)$and a sequence $\left(\varphi_{k} \in \mathcal{D}\right)_{k \in \mathbb{N}}$ 
converge to $f$ in $L^{2}\left(\mathbb{R}_{+}\right)$as $k \rightarrow \infty$ (as is well known (e.g., [3]), $\mathcal{D}$ is a dense set in $L^{2}\left(\mathbb{R}_{+}\right)$). One can show $[4,5,17,23]$ that $\langle\circ, f\rangle:=\left(L^{2}\right)-\lim _{k \rightarrow \infty}\left\langle 0, \varphi_{k}\right\rangle$ (i.e. the limit in the topology of the space $\left.\left(L^{2}\right)\right)$ is well-defined as an element of $\left(L^{2}\right)$.

Denote by $1_{A}$ the indicator of a set $A$. Set $1_{[0,0)} \equiv 0$ and consider $\left\langle 0,1_{[0, t)}\right\rangle \in\left(L^{2}\right), t \in \mathbb{R}_{+}$. It follows from (1) and (3) that $\left(\left\langle 0,1_{[0, t)}\right\rangle_{t \in \mathbb{R}_{+}}\right)$can be identified with a Lévy process on the probability space $\left(\mathcal{D}^{\prime}, \mathcal{C}\left(\mathcal{D}^{\prime}\right), \mu\right)$ (see, e.g., $\left.[4,5]\right)$. So, one can write $L_{t}=\left\langle 0,1_{[0, t)}\right\rangle \in\left(L^{2}\right)$.

\subsection{Lytvynov's generalization of the CRP}

Denote by $\widehat{\otimes}$ a symmetric tensor product, by a subscript $\mathbb{C}$-complexifications of spaces. Set $\mathbb{Z}_{+}:=\mathbb{N} \cup\{0\}$. Denote by $\mathcal{P}$ the set of complex-valued polynomials on $\mathcal{D}^{\prime}$ that consists of zero and elements of the form

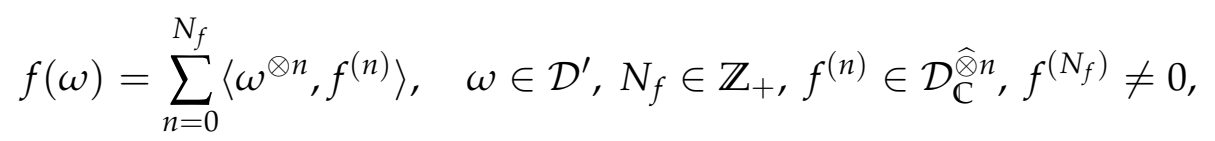

here $N_{f}$ is called the power of a polynomial $f ;\left\langle\omega^{\otimes 0}, f^{(0)}\right\rangle:=f^{(0)} \in \mathcal{D}_{\mathbb{C}}^{\widehat{\otimes} 0}:=\mathbb{C}$. The measure $\mu$ of a Lévy white noise has a holomorphic at zero Laplace transform (this follows from (3) and properties of the measure $v$, see also [23]), therefore $\mathcal{P}$ is a dense set in $\left(L^{2}\right)$ [26]. Denote by $\mathcal{P}_{n}$ the set of polynomials of power smaller or equal to $n$, by $\overline{\mathcal{P}}_{n}$ the closure of $\mathcal{P}_{n}$ in $\left(L^{2}\right)$. Let for $n \in \mathbb{N} \mathbf{P}_{n}:=\overline{\mathcal{P}}_{n} \ominus \overline{\mathcal{P}}_{n-1}$ (the orthogonal difference in $\left(L^{2}\right)$ ), $\mathbf{P}_{0}:=\overline{\mathcal{P}}_{0}$. It is clear that

$$
\left(L^{2}\right)=\bigoplus_{n=0}^{\infty} \mathbf{P}_{n} .
$$

Let $f^{(n)} \in \mathcal{D}_{\mathbb{C}^{\widehat{\otimes}} n}, n \in \mathbb{Z}_{+}$. Denote by $:\left\langle 0^{\otimes n}, f^{(n)}\right\rangle:$ the orthogonal projection of a monomial $\left\langle 0^{\otimes n}, f^{(n)}\right\rangle$ onto $\mathbf{P}_{n}$. Let us define real (i.e. bilinear) scalar products $(\cdot, \cdot)_{\text {ext }}$ on $\mathcal{D}_{\mathbf{C}}^{\widehat{\otimes} n}, n \in \mathbb{Z}_{+}$, by setting for $f^{(n)}, g^{(n)} \in \mathcal{D}_{\mathbf{C}}^{\widehat{\otimes} n}$

$$
\left(f^{(n)}, g^{(n)}\right)_{e x t}:=\frac{1}{n !} \int_{\mathcal{D}^{\prime}}:\left\langle\omega^{\otimes n}, f^{(n)}\right\rangle::\left\langle\omega^{\otimes n}, g^{(n)}\right\rangle: \mu(d \omega) .
$$

This definition is well posed: it is clear that $(\cdot, \cdot)_{\text {ext }}$ are quasiscalar products on $\mathcal{D}_{\mathrm{C}}^{\widehat{\otimes} n}$, the fact that these products are scalar follows from their explicit formula calculated in [23] (see formula (6) below).

By $|\cdot|_{\text {ext }}$ we denote the norms corresponding to scalar products (4), i.e.

$$
\left|f^{(n)}\right|_{\text {ext }}:=\sqrt{\left(f^{(n)}, \overline{f^{(n)}}\right)_{e x t}}
$$

Denote by $\mathcal{H}_{\text {ext }}^{(n)}, n \in \mathbb{Z}_{+}$, the completions of $\mathcal{D}_{\mathrm{C}}^{\widehat{\otimes} n}$ with respect to the norms $|\cdot|_{\text {ext }}$. For $F^{(n)} \in \mathcal{H}_{\text {ext }}^{(n)}$ define a Wick monomial $:\left\langle 0^{\otimes n}, F^{(n)}\right\rangle: \stackrel{\text { def }}{=}\left(L^{2}\right)-\lim _{k \rightarrow \infty}:\left\langle 0^{\otimes n}, f_{k}^{(n)}\right\rangle:$, where $\mathcal{D}_{\mathrm{C}}^{\widehat{\otimes} n} \ni f_{k}^{(n)} \rightarrow F^{(n)}$ as $k \rightarrow \infty$ in $\mathcal{H}_{\text {ext }}^{(n)}$ (the well-posedness of this definition can be proved by the method of "mixed sequences"). Since, as is easy to see, for each $n \in \mathbb{Z}_{+}$the set $\left\{:\left\langle 0^{\otimes n}, f^{(n)}\right\rangle: \mid f^{(n)} \in \mathcal{D}_{\mathrm{C}}^{\widehat{\otimes} n}\right\}$ is dense in $\mathbf{P}_{n}, F \in\left(L^{2}\right)$ if and only if there exists a unique sequence of kernels $F^{(n)} \in \mathcal{H}_{\text {ext }}^{(n)}, n \in \mathbb{Z}_{+}$, such that

$$
F=\sum_{n=0}^{\infty}:\left\langle 0^{\otimes n}, F^{(n)}\right\rangle:
$$


(the series converges in $\left(L^{2}\right)$ ) and

$$
\|F\|_{\left(L^{2}\right)}^{2}=\int_{\mathcal{D}^{\prime}}|F(\omega)|^{2} \mu(d \omega)=\mathbb{E}|F|^{2}=\sum_{n=0}^{\infty} n !\left|F^{(n)}\right|_{\text {ext }}^{2}<\infty .
$$

So, for $F, G \in\left(L^{2}\right)$ the real scalar product has a form

$$
(F, G)_{\left(L^{2}\right)}=\int_{\mathcal{D}^{\prime}} F(\omega) G(\omega) \mu(d \omega)=\mathbb{E}[F G]=\sum_{n=0}^{\infty} n !\left(F^{(n)}, G^{(n)}\right)_{\text {ext }}
$$

where $F^{(n)}, G^{(n)} \in \mathcal{H}_{\text {ext }}^{(n)}$ are the kernels from decompositions (5) for $F$ and $G$ respectively. In particular, for $F^{(n)} \in \mathcal{H}_{\text {ext }}^{(n)}$ and $G^{(m)} \in \mathcal{H}_{\text {ext }}^{(m)}, n, m \in \mathbb{Z}_{+}$,

$$
\begin{aligned}
\left(:\left\langle 0^{\otimes n}, F^{(n)}\right\rangle:,:\left\langle 0^{\otimes m}, G^{(m)}\right\rangle:\right)_{\left(L^{2}\right)} & =\int_{\mathcal{D}^{\prime}}:\left\langle\omega^{\otimes n}, F^{(n)}\right\rangle::\left\langle\omega^{\otimes m}, G^{(m)}\right\rangle: \mu(d \omega) \\
& =\mathbb{E}\left[:\left\langle 0^{\otimes n}, F^{(n)}\right\rangle::\left\langle 0^{\otimes m}, G^{(m)}\right\rangle:\right]=\delta_{n, m} n !\left(F^{(n)}, G^{(n)}\right)_{\text {ext }} .
\end{aligned}
$$

Also we note that in the space $\left(L^{2}\right):\left\langle 0^{\otimes 0}, F^{(0)}\right\rangle:=\left\langle 0^{\otimes 0}, F^{(0)}\right\rangle=F^{(0)}$ and $:\left\langle 0, F^{(1)}\right\rangle:=\left\langle 0, F^{(1)}\right\rangle$ [23].

In what follows, we need an explicit formula for the scalar products $(\cdot, \cdot)_{\text {ext }}$. Let us write out this formula. Denote by $\|\cdot\|_{v}$ the norm in the space $L^{2}(\mathbb{R}, v)$ of (classes of) square integrable with respect to the Lévy measure $v$ (see (1)) real-valued functions on $\mathbb{R}$. Let

$$
p_{n}(x):=x^{n}+a_{n, n-1} x^{n-1}+\cdots+a_{n, 1} x, \quad a_{n, j} \in \mathbb{R}, j \in\{1, \ldots, n-1\}, n \in \mathbb{N},
$$

be polynomials orthogonal in $L^{2}(\mathbb{R}, v)$, i.e. for natural numbers $n, m$ such that $n \neq m$, $\int_{\mathbb{R}} p_{n}(x) p_{m}(x) v(d x)=0$. Then, as it follows from [23], for $F^{(n)}, G^{(n)} \in \mathcal{H}_{\text {ext }}^{(n)}, n \in \mathbb{N}$,

$$
\begin{aligned}
& \left(F^{(n)}, G^{(n)}\right)_{e x t} \equiv\left(F^{(n)}, G^{(n)}\right)_{\mathcal{H}_{e x t}^{(n)}} \\
& =\sum_{\substack{k, l_{j}, s_{j} \in \mathbb{N}: \\
l_{1} s_{1}+\cdots+, l_{k} s_{k}=n}} \frac{n !}{s_{1} ! \cdots>s_{k} !}\left(\frac{\left\|p_{l_{1}}\right\|_{v}}{l_{1} !}\right)^{2 s_{1}} \cdots\left(\frac{\left\|p_{l_{k}}\right\|_{v}}{l_{k} !}\right)^{2 s_{k}} \\
& \times \int_{\mathbb{R}_{+}^{s_{1}+\cdots+s_{k}}} F^{(n)}(\underbrace{u_{1}, \ldots, u_{1}}_{l_{1}}, \ldots, \underbrace{u_{s_{1}}, \ldots, u_{s_{1}}}_{l_{1}}, \ldots, \underbrace{u_{s_{1}+\cdots+s_{k}}, \ldots, u_{s_{1}+\cdots+s_{k}}}_{l_{k}}) \\
& \times G^{(n)}(\underbrace{u_{1}, \ldots, u_{1}}_{l_{1}}, \ldots, \underbrace{u_{s_{1}}, \ldots, u_{s_{1}}}_{l_{1}}, \ldots, \underbrace{u_{s_{1}+\cdots+s_{k}}, \ldots, u_{s_{1}+\cdots+s_{k}}}_{l_{k}}) d u_{1} \cdots d u_{s_{1}+\cdots+s_{k}} .
\end{aligned}
$$

In particular, for $n=1$

$$
\left(F^{(1)}, G^{(1)}\right)_{\text {ext }} \equiv\left(F^{(1)}, G^{(1)}\right)_{\mathcal{H}_{\text {ext }}^{(1)}}=\left\|p_{1}\right\|_{v}^{2} \int_{\mathbb{R}_{+}} F^{(1)}(u) G^{(1)}(u) d u=\left(F^{(1)}, G^{(1)}\right)_{L^{2}\left(\mathbb{R}_{+}\right)_{\mathrm{C}}}
$$

(by (2) $\left\|p_{1}\right\|_{v}^{2}=\int_{\mathbb{R}} x^{2} v(d x)=1$ ); in the case $n=2$ we have

$$
\begin{aligned}
& \left(F^{(2)}, G^{(2)}\right)_{\text {ext }} \equiv\left(F^{(2)}, G^{(2)}\right)_{\mathcal{H}_{\text {ext }}^{(2)}}=\left\|p_{1}\right\|_{v}^{4} \int_{\mathbb{R}_{+}^{2}} F^{(2)}\left(u_{1}, u_{2}\right) G^{(2)}\left(u_{1}, u_{2}\right) d u_{1} d u_{2} \\
& +\frac{\left\|p_{2}\right\|_{v}^{2}}{2} \int_{\mathbb{R}_{+}} F^{(2)}(u, u) G^{(2)}(u, u) d u=\left(F^{(2)}, G^{(2)}\right)_{L^{2}\left(\mathbb{R}_{+}\right)_{\mathrm{C}}^{\otimes 2}}+\frac{\left\|p_{2}\right\|_{v}^{2}}{2} \int_{\mathbb{R}_{+}} F^{(2)}(u, u) G^{(2)}(u, u) d u,
\end{aligned}
$$

etc. 
Remark. Note that the explicit formula for scalar products in $\mathcal{H}_{\text {ext }}^{(n)}, n \in \mathbb{Z}_{+}$, calculated in [23], differs from (6). But it is very easy to verify that actually these formulas differ by the record form only.

Denote $\mathcal{H}:=L^{2}\left(\mathbb{R}_{+}\right)$, then $\mathcal{H}_{\mathbb{C}}=L^{2}\left(\mathbb{R}_{+}\right)_{\mathbb{C}}$ (in what follows, this notation will be used very often). It follows from (7) that $\mathcal{H}_{\text {ext }}^{(1)}=\mathcal{H}_{\mathbb{C}}$; and, as is easily seen, for $n \in \mathbb{N} \backslash\{1\}$ one can identify $\mathcal{H}_{\mathrm{C}}^{\widehat{\otimes} n}$ with the proper subspace of $\mathcal{H}_{\text {ext }}^{(n)}$ that consists of "vanishing on diagonals" elements (roughly speaking, such that $F^{(n)}\left(u_{1}, \ldots, u_{n}\right)=0$ if there exist $k, j \in\{1, \ldots, n\}: k \neq j$, but $u_{k}=u_{j}$ ). In this sense the space $\mathcal{H}_{\text {ext }}^{(n)}$ is an extension of $\mathcal{H}_{\mathbb{C}}^{\widehat{\otimes} n}$ (this explains why we use the subscript "ext" in our designations).

\subsection{A regular rigging of $\left(L^{2}\right)$}

Denote $\mathcal{P}_{W}:=\left\{f=\sum_{n=0}^{N_{f}}:\left\langle 0^{\otimes n}, f^{(n)}\right\rangle:, f^{(n)} \in \mathcal{D}_{\mathrm{C}}^{\otimes} n, N_{f} \in \mathbb{Z}_{+}\right\} \subset\left(L^{2}\right)$. Accept on default $\beta \in[0,1], q \in \mathbb{Z}$ in the case $\beta \in(0,1]$ and $q \in \mathbb{Z}_{+}$if $\beta=0$. Define real (bilinear) scalar products $(\cdot, \cdot)_{q, \beta}$ on $\mathcal{P}_{W}$ by setting for

$$
\begin{aligned}
& f=\sum_{n=0}^{N_{f}}:\left\langle 0^{\otimes n}, f^{(n)}\right\rangle:, g=\sum_{n=0}^{N_{g}}:\left\langle o^{\otimes n}, g^{(n)}\right\rangle: \in \mathcal{P}_{W} \\
& (f, g)_{q, \beta}:=\sum_{n=0}^{\min \left(N_{f}, N_{g}\right)}(n !)^{1+\beta} 2^{q n}\left(f^{(n)}, g^{(n)}\right)_{\text {ext }} .
\end{aligned}
$$

It is easy to verify that the axioms of a scalar product are fulfilled. In particular, if $(f, \bar{f})_{q, \beta}=0$ then $f=0$ in $\left(L^{2}\right)$. In fact, $(f, \bar{f})_{q, \beta}=\sum_{n=0}^{N_{f}}(n !)^{1+\beta_{2}{ }^{q n}}\left|f^{(n)}\right|_{\text {ext }}^{2}=0$ if and only if $\left|f^{(n)}\right|_{\text {ext }}^{2}=0$ for each $n \in\left\{0, \ldots, N_{f}\right\}$, so $\|f\|_{\left(L^{2}\right)}^{2}=\sum_{n=0}^{N_{f}} n !\left|f^{(n)}\right|_{\text {ext }}^{2}=0$.

Let $\|\cdot\|_{q, \beta}$ be the norms corresponding to scalar products $(\cdot, \cdot)_{q, \beta}$, i.e. $\|f\|_{q, \beta}=\sqrt{(f, \bar{f})_{q, \beta}}$. Denote by $\left(L^{2}\right)_{q}^{\beta}$ the completions of $\mathcal{P}_{W}$ with respect to these norms; and set $\left(L^{2}\right)^{\beta}:=\underset{q \rightarrow+\infty}{\operatorname{pr} \lim _{q}}\left(L^{2}\right)_{q}^{\beta}$ (the projective limit of spaces, i.e. $\left(L^{2}\right)^{\beta}=\bigcap_{q}\left(L^{2}\right)_{q}^{\beta}$ provided by the projective limit topology, see, e.g., [2,3] for details).

Definition 2. The spaces $\left(L^{2}\right)_{q}^{\beta}$ and $\left(L^{2}\right)^{\beta}$ are called parametrized Kondratiev-type spaces of regular test functions.

As is easy to see, $F \in\left(L^{2}\right)_{q}^{\beta}$ if and only if $F$ can be uniquely presented as series (5) (with kernels $\left.F^{(n)} \in \mathcal{H}_{\text {ext }}^{(n)}\right)$ that converges in $\left(L^{2}\right)_{q}^{\beta}$, and

$$
\|F\|_{q, \beta}^{2}:=\|F\|_{\left(L^{2}\right)_{q}^{\beta}}^{2}=\sum_{n=0}^{\infty}(n !)^{1+\beta_{2}{ }^{q n}}\left|F^{(n)}\right|_{e x t}^{2}<\infty .
$$

Further, it is clear that for $F, G \in\left(L^{2}\right)_{q}^{\beta}$ the real scalar product has a form

$$
(F, G)_{\left(L^{2}\right)_{q}^{\beta}}=\sum_{n=0}^{\infty}(n !)^{1+\beta_{2}^{q n}}\left(F^{(n)}, G^{(n)}\right)_{\text {ext }},
$$

where $F^{(n)}, G^{(n)} \in \mathcal{H}_{\text {ext }}^{(n)}$ are the kernels from decompositions (5) for $F$ and $G$ respectively. Finally, $F \in\left(L^{2}\right)^{\beta}$ if and only if $F$ can be uniquely presented in form (5) and series (8) converges for each $q \in \mathbb{Z}_{+}$. 
Proposition ( [16]). For any $\beta \in(0,1]$ and any $q \in \mathbb{Z}$, in the same way as for $\beta=0$ and any $q \in \mathbb{Z}_{+}$, the space $\left(L^{2}\right)_{q}^{\beta}$ is densely and continuously embedded into $\left(L^{2}\right)$. $\left.\left(L^{2}\right)\right)$

Taking into account this result, we can consider a chain (a parametrized regular rigging of

$$
\left(L^{2}\right)^{-\beta} \supset\left(L^{2}\right)_{-q}^{-\beta} \supset\left(L^{2}\right) \supset\left(L^{2}\right)_{q}^{\beta} \supset\left(L^{2}\right)^{\beta},
$$

where $\left(L^{2}\right)_{-q}^{-\beta}$ and $\left(L^{2}\right)^{-\beta}=$ ind $\lim _{q \rightarrow+\infty}\left(L^{2}\right)_{-q}^{-\beta}$ (the inductive limit of spaces, i.e. $\left(L^{2}\right)^{-\beta}=$ $\bigcup_{q}\left(L^{2}\right)_{-q}^{-\beta}$ provided by the inductive limit topology, see, e.g., $[2,3]$ for details) are the spaces dual of $\left(L^{2}\right)_{q}^{\beta}$ and $\left(L^{2}\right)^{\beta}$ respectively.

Definition 3. The spaces $\left(L^{2}\right)_{-q}^{-\beta}$ and $\left(L^{2}\right)^{-\beta}$ are called parametrized Kondratiev-type spaces of regular generalized functions. lows.

The following statement from the definition of $\left(L^{2}\right)_{-q}^{-\beta}$ and the general duality theory fol-

Proposition. 1) Any regular generalized function $F \in\left(L^{2}\right)_{-q}^{-\beta}$ can be uniquely presented as formal series (5) (with kernels $\left.F^{(n)} \in \mathcal{H}_{\text {ext }}^{(n)}\right)$ that converges in $\left(L^{2}\right)_{-q}^{-\beta}$, and

$$
\|F\|_{-q,-\beta}^{2}:=\|F\|_{\left(L^{2}\right)_{-q}^{-\beta}}^{2}=\sum_{n=0}^{\infty}(n !)^{1-\beta} 2^{-q n}\left|F^{(n)}\right|_{e x t}^{2}<\infty .
$$

Vice versa, any formal series (5) such that series (10) converges, is a regular generalized function from $\left(L^{2}\right)_{-q}^{-\beta}$ (i.e. now series (5) converges in $\left(L^{2}\right)_{-q}^{-\beta}$ ).

2) For $F, G \in\left(L^{2}\right)_{-q}^{-\beta}$ the real scalar product has a form

$$
(F, G)_{\left(L^{2}\right)_{-q}^{-\beta}}=\sum_{n=0}^{\infty}(n !)^{1-\beta} 2^{-q n}\left(F^{(n)}, G^{(n)}\right)_{\text {ext }}
$$

where $F^{(n)}, G^{(n)} \in \mathcal{H}_{\text {ext }}^{(n)}$ are the kernels from decompositions (5) for $F$ and $G$ respectively.

3) The dual pairing between $F \in\left(L^{2}\right)_{-q}^{-\beta}$ and $f \in\left(L^{2}\right)_{q}^{\beta}$ that is generated by the scalar product in $\left(L^{2}\right)$, has a form

$$
\langle\langle F, f\rangle\rangle_{\left(L^{2}\right)}=\sum_{n=0}^{\infty} n !\left(F^{(n)}, f^{(n)}\right)_{e x t}
$$

where $F^{(n)}, f^{(n)} \in \mathcal{H}_{\text {ext }}^{(n)}$ are the kernels from decompositions (5) for $F$ and $f$ respectively.

4) $F \in\left(L^{2}\right)^{-\beta}$ if and only if $F$ can be uniquely presented in form (5) and norm (10) is finite for some $q \in \mathbb{Z}_{+}$.

Note that the term "regular generalized functions" is connected with the fact that the kernels from decompositions (5) for elements of positive and negative spaces of chain (9) belong to the same spaces $\mathcal{H}_{\text {ext }}^{(n)}$. 


\subsection{Stochastic integration and differentiation}

In this subsection it will be convenient to denote the spaces $\left(L^{2}\right)_{q}^{\beta},\left(L^{2}\right)=\left(L^{2}\right)_{0}^{0}$ and $\left(L^{2}\right)_{-q}^{-\beta}$ from chain (9) by $\left(L^{2}\right)_{q}^{\beta}, \beta \in[-1,1], q \in \mathbb{Z}$. The norms in these spaces are given, obviously, by formula (8) (cf. (8) and (10)).

Decomposition (5) for elements of $\left(L^{2}\right)_{q}^{\beta}$ defines an isometric isomorphism (a generalized Wiener-Itô-Sigal isomorphism)

$$
\mathbf{I}:\left(L^{2}\right)_{q}^{\beta} \rightarrow \bigoplus_{n=0}^{\infty}(n !)^{1+\beta} 2^{q n} \mathcal{H}_{\text {ext }}^{(n)},
$$

where $\bigoplus_{n=0}^{\infty}(n !)^{1+\beta} 2^{q n} \mathcal{H}_{\text {ext }}^{(n)}$ is a weighted extended symmetric Fock space (cf. [20]): for $F \in$ $\left(L^{2}\right)_{q}^{\beta}$ of form $(5) \mathbf{I} F=\left(F^{(0)}, F^{(1)}, \ldots\right) \in \bigoplus_{n=0}^{\infty}(n !)^{1+\beta_{2}{ }^{q n}} \mathcal{H}_{\text {ext }}^{(n)}$. Let $\mathbf{1}: \mathcal{H}_{\mathbb{C}} \rightarrow \mathcal{H}_{\mathbb{C}}$ be the identity operator. Then the operator I $\otimes \mathbf{1}:\left(L^{2}\right)_{q}^{\beta} \otimes \mathcal{H}_{\mathbf{C}} \rightarrow\left(\underset{n=0}{\infty}(n !)^{1+\beta_{2}{ }^{q n}} \mathcal{H}_{\text {ext }}^{(n)}\right) \otimes \mathcal{H}_{\mathbb{C}} \cong$ $\bigoplus_{n=0}^{\infty}(n !)^{1+\beta_{2} 2^{q n}}\left(\mathcal{H}_{\text {ext }}^{(n)} \otimes \mathcal{H}_{\mathbb{C}}\right)$ is an isometric isomorphism between the spaces $\left(L^{2}\right)_{q}^{\beta} \otimes \mathcal{H}_{\mathbb{C}}$ and $\bigoplus_{n=0}^{\infty}(n !)^{1+\beta_{2} 2^{q n}}\left(\mathcal{H}_{\text {ext }}^{(n)} \otimes \mathcal{H}_{\mathrm{C}}\right)$. It is obvious that for arbitrary $m \in \mathbb{Z}_{+}$and $F^{(m)} \in \mathcal{H}_{\text {ext }}^{(m)} \otimes \mathcal{H}_{\mathrm{C}}$ a vector $(\underbrace{0, \ldots, 0}_{m}, F^{(m)}, 0, \ldots)$ belongs to $\bigoplus_{n=0}^{\infty}(n !)^{1+\beta_{2}{ }^{q n}}\left(\mathcal{H}_{\text {ext }}^{(n)} \otimes \mathcal{H}_{\mathbb{C}}\right)$. Set

$$
:\left\langle 0^{\otimes m}, F^{(m)}\right\rangle: \stackrel{\text { def }}{=}(\mathbf{I} \otimes \mathbf{1})^{-1}(\underbrace{0, \ldots, 0}_{m}, F^{(m)}, 0, \ldots) \in\left(L^{2}\right)_{q}^{\beta} \otimes \mathcal{H}_{\mathbf{C}} .
$$

It is clear that elements : $\left\langle 0^{\otimes n}, F^{(n)}\right\rangle:, n \in \mathbb{Z}_{+}$, form orthogonal bases in the spaces $\left(L^{2}\right)_{q}^{\beta} \otimes \mathcal{H}_{\mathbb{C}}$ in the sense that any $F \in\left(L^{2}\right)_{q}^{\beta} \otimes \mathcal{H}_{\mathbb{C}}$ can be uniquely presented as

$$
F(\cdot)=\sum_{n=0}^{\infty}:\left\langle 0^{\otimes n}, F^{(n)}\right\rangle:, \quad F^{(n)} \in \mathcal{H}_{\text {ext }}^{(n)} \otimes \mathcal{H}_{\mathrm{C}}
$$

(the series converges in $\left.\left(L^{2}\right)_{q}^{\beta} \otimes \mathcal{H}_{\mathbb{C}}\right)$, with

$$
\|F\|_{\left(L^{2}\right)_{q}^{\beta} \otimes \mathcal{H}_{C}}^{2}=\sum_{n=0}^{\infty}(n !)^{1+\beta} 2^{q n}\left|F^{(n)}\right|_{\mathcal{H}_{e x t}^{(n)} \otimes \mathcal{H}_{C}}^{2}<\infty .
$$

Let us describe the construction of an extended stochastic integral that is based on decomposition (11) (a detailed presentation is given in [16,17]). Let $F^{(n)} \in \mathcal{H}_{\text {ext }}^{(n)} \otimes \mathcal{H}_{\mathbb{C}}, n \in \mathbb{N}$. We select a representative (a function) $\dot{f}^{(n)} \in F^{(n)}$ such that

$$
\dot{f}_{u}^{(n)}\left(u_{1}, \ldots, u_{n}\right)=0 \text { if for some } \mathrm{k} \in\{1, \ldots, \mathrm{n}\} u=u_{k} .
$$

Accept on default $\Delta \in \mathcal{B}\left(\mathbb{R}_{+}\right)$(we remind that $\mathcal{B}$ denotes the Borel $\sigma$-algebra). Let $\widehat{f}_{\Delta}^{(n)}$ be the symmetrization of a function $\dot{f}^{(n)} 1_{\Delta}(\cdot)$ by $n+1$ variables. Define $\widehat{F}_{\Delta}^{(n)} \in \mathcal{H}_{\text {ext }}^{(n+1)}$ as the equivalence class in $\mathcal{H}_{\text {ext }}^{(n+1)}$ generated by $\widehat{f}_{\Delta}^{(n)}$ (i.e. $\widehat{f}_{\Delta}^{(n)} \in \widehat{F}_{\Delta}^{(n)}$ ). It is proved in [16,17] that this definition is well-posed (in particular, $\widehat{F}_{\Delta}^{(n)}$ does not depend on a choice of a representative $\dot{f}^{(n)} \in F^{(n)}$ satisfying (12)) and $\left|\widehat{F}_{\Delta}^{(n)}\right|_{\text {ext }} \leq\left|F^{(n)} 1_{\Delta}(\cdot)\right|_{\mathcal{H}_{\text {ext }}^{(n)} \otimes \mathcal{H}_{\mathrm{C}}} \leq\left|F^{(n)}\right|_{\mathcal{H}_{\text {ext }}^{(n)} \otimes \mathcal{H}_{\mathrm{C}}}$. 
Definition 4. We define the extended stochastic integral with respect to a Lévy process $L$

$$
\int_{\Delta} \circ(u) \widehat{d} L_{u}:\left(L^{2}\right)_{q}^{\beta} \otimes \mathcal{H}_{\mathbb{C}} \rightarrow\left(L^{2}\right)_{q-1}^{\beta}
$$

by a formula

$$
\int_{\Delta} F(u) \widehat{d} L_{u}:=\sum_{n=0}^{\infty}:\left\langle\diamond^{\otimes n+1}, \widehat{F}_{\Delta}^{(n)}\right\rangle:
$$

where $\widehat{F}_{\Delta}^{(0)}:=F^{(0)} 1_{\Delta}(\cdot) \in \mathcal{H}_{\mathbb{C}}=\mathcal{H}_{\text {ext }}^{(1)}$, and $\widehat{F}_{\Delta}^{(n)} \in \mathcal{H}_{\text {ext }}^{(n+1)}, n \in \mathbb{N}$, are constructed by the kernels $F^{(n)} \in \mathcal{H}_{\text {ext }}^{(n)} \otimes \mathcal{H}_{\mathbb{C}}$ from decomposition (11) for $F$.

One can show quite analogously to [16] that this integral is a linear continuous operator; and, moreover, if $F$ is integrable by Itô then $F$ is integrable in the extended sense and the extended stochastic integral coincides with the Itô stochastic integral.

Sometimes it can be convenient to define the extended stochastic integral by formula (14) as a linear operator

$$
\int_{\Delta} \circ(u) \widehat{d} L_{u}:\left(L^{2}\right)_{q}^{\beta} \otimes \mathcal{H}_{\mathrm{C}} \rightarrow\left(L^{2}\right)_{q}^{\beta} .
$$

If $\beta=-1$ then this operator is continuous (bounded) [16], for $\beta \in(-1,1]$ operator (15) is unbounded. But if we accept the set

$$
\left\{F \in\left(L^{2}\right)_{q}^{\beta} \otimes \mathcal{H}_{\mathbb{C}}:\left\|\int_{\Delta} F(u) \widehat{d} L_{u}\right\|_{q, \beta}^{2}=\left.\sum_{n=0}^{\infty}((n+1) !)^{1+\beta_{2}{ }^{q(n+1)} \mid \widehat{F}_{\Delta}^{(n)}}\right|_{\text {ext }} ^{2}<\infty\right\}
$$

as the domain of integral (15) then the last is a closed operator [16]. Also we note that the extended stochastic integral can be defined by formula (14) as a linear continuous operator acting from $\left(L^{2}\right)^{\beta} \otimes \mathcal{H}_{\mathbb{C}}:=\operatorname{pr} \lim _{q \rightarrow+\infty}\left(L^{2}\right)_{q}^{\beta} \otimes \mathcal{H}_{\mathbf{C}}$ to $\left(L^{2}\right)^{\beta}$, or from $\left(L^{2}\right)^{-\beta} \otimes \mathcal{H}_{\mathbb{C}}:=$ ind $\lim _{q \rightarrow+\infty}\left(L^{2}\right)_{-q}^{-\beta} \otimes \mathcal{H}_{\mathbb{C}}$ to $\left(L^{2}\right)^{-\beta}$, here $\beta \in[0,1]$.

Now for plenitude of picture we recall very briefly a notion of a Hida stochastic derivative in the Lévy white noise analysis, in terms of Lytvynov's CRP (see $[8,16,17]$ for a detailed presentation).

Definition 5. We define the Hida stochastic derivative $1_{\Delta}(\cdot) \partial .:\left(L^{2}\right)_{1-q}^{-\beta} \rightarrow\left(L^{2}\right)_{-q}^{-\beta} \otimes \mathcal{H}_{\mathrm{C}}$ as a linear continuous operator adjoint to extended stochastic integral (13), i.e. for all $F \in\left(L^{2}\right)_{q}^{\beta} \otimes$ $\mathcal{H}_{\mathrm{C}}$ and $G \in\left(L^{2}\right)_{1-q}^{-\beta}$

$$
\left\langle\left\langle F(\cdot), 1_{\Delta}(\cdot) \partial . G\right\rangle\right\rangle_{\left(L^{2}\right) \otimes \mathcal{H}_{\mathrm{C}}}=\left\langle\left\langle\int_{\Delta} F(u) \widehat{d} L_{u}, G\right\rangle\right\rangle_{\left(L^{2}\right)} .
$$

If instead of integral (13) one uses integral (15), the corresponding Hida stochastic derivative will be a linear unbounded (except the case $\beta=-1$ ), but closed operator acting from $\left(L^{2}\right)_{-q}^{-\beta}$ to $\left(L^{2}\right)_{-q}^{-\beta} \otimes \mathcal{H}_{\mathrm{C}}$ [8]. Further, it is clear that the Hida stochastic derivative can be defined as a linear continuous operator acting from $\left(L^{2}\right)^{\beta}$ to $\left(L^{2}\right)^{\beta} \otimes \mathcal{H}_{\mathbb{C}}(\beta \in[-1,1])$ that is adjoint to the corresponding extended stochastic integral. We note also that the extended stochastic integral and the Hida stochastic derivative are mutually adjoint operators [8, 16, 17].

Let us write out an explicit formula for the Hida stochastic derivative in terms of decompositions by the Wick monomials. Let $G^{(n)} \in \mathcal{H}_{\text {ext }}^{(n)}, n \in \mathbb{N}, \dot{g}^{(n)} \in G^{(n)}$ be a representative of $G^{(n)}$. 
We consider $\dot{g}^{(n)}(\cdot)$, i.e. separate a one argument of $\dot{g}^{(n)}$, and define $G^{(n)}(\cdot) \in \mathcal{H}_{\text {ext }}^{(n-1)} \otimes \mathcal{H}_{\mathbb{C}}$ as the equivalence class in $\mathcal{H}_{\text {ext }}^{(n-1)} \otimes \mathcal{H}_{\mathbb{C}}$ generated by $\dot{g}^{(n)}(\cdot)$ (i.e. $\dot{g}^{(n)}(\cdot) \in G^{(n)}(\cdot)$ ). It is proved in [17] that this definition is well-posed (in particular, $G^{(n)}(\cdot)$ does not depend on a choice of a representative $\left.\dot{g}^{(n)} \in G^{(n)}\right)$ and

$$
\left|G^{(n)}(\cdot)\right|_{\mathcal{H}_{\text {ext }}^{(n-1)} \otimes \mathcal{H}_{\mathrm{C}}} \leq\left|G^{(n)}\right|_{\text {ext }}
$$

Note that, in spite of estimate (16), the space $\mathcal{H}_{\text {ext }}^{(n)}, n \in \mathbb{N} \backslash\{1\}$, is not a subspace of $\mathcal{H}_{\text {ext }}^{(n-1)} \otimes$ $\mathcal{H}_{\mathbb{C}}$ because different elements of $\mathcal{H}_{\text {ext }}^{(n)}$ can coincide as elements of $\mathcal{H}_{\text {ext }}^{(n-1)} \otimes \mathcal{H}_{\mathbb{C}}$.

The following statement easily follows from results of $[8,16,17]$.

Proposition. For a test or square integrable or generalized function $G$ of form (5)

$$
1_{\Delta}(\cdot) \partial . G=\sum_{n=1}^{\infty} n:\left\langle 0^{\otimes n-1}, G^{(n)}(\cdot) 1_{\Delta}(\cdot)\right\rangle: \equiv \sum_{n=0}^{\infty}(n+1):\left\langle 0^{\otimes n}, G^{(n+1)}(\cdot) 1_{\Delta}(\cdot)\right\rangle: .
$$

At last, we recall a notion of operators of stochastic differentiation (see [6,7] for a detailed presentation). Let $n, m \in \mathbb{Z}_{+}$. Consider a function $h: \mathbb{R}_{+}^{n+m} \rightarrow \mathbb{C}$. Denote

$$
\begin{aligned}
& \widetilde{h}\left(u_{1}, \ldots, u_{n} ; u_{n+1}, \ldots, u_{n+m}\right) \\
& :=\left\{\begin{array}{l}
h\left(u_{1}, \ldots, u_{n+m}\right), \text { if for all } i \in\{1, \ldots, n\}, j \in\{n+1, \ldots, n+m\} u_{i} \neq u_{j} \\
0, \text { in other cases }
\end{array}\right.
\end{aligned}
$$

Let $F^{(n)} \in \mathcal{H}_{\text {ext }}^{(n)}, G^{(m)} \in \mathcal{H}_{\text {ext }}^{(m)}$. We select representatives (functions) $\dot{f}^{(n)} \in F^{(n)}$ and $\dot{g}^{(m)} \in G^{(m)}$. Set $h\left(u_{1}, \ldots, u_{n+m}\right):=\dot{f}^{(n)}\left(u_{1}, \ldots, u_{n}\right) \cdot \dot{g}^{(m)}\left(u_{n+1}, \ldots, u_{n+m}\right)$. Let $\widehat{f^{(n)} g^{(m)}}$ be the symmetrization of $\widetilde{h}$ (see (17)) by all variables, $F^{(n)} \diamond G^{(m)} \in \mathcal{H}_{\text {ext }}^{(n+m)}$ be the equivalence class in $\mathcal{H}_{\text {ext }}^{(n+m)}$ that is generated by $\widehat{f^{(n)} g^{(m)}}$ (i.e. $\widehat{f^{(n)} g^{(m)}} \in F^{(n)} \diamond G^{(m)}$ ). It is proved in [6] that this definition is well-posed (in particular, $F^{(n)} \diamond G^{(m)}$ does not depend on a choice of representatives from $F^{(n)}$ and $G^{(m)}$ ) and

$$
\left|F^{(n)} \diamond G^{(m)}\right|_{\text {ext }} \leq\left|F^{(n)}\right|_{\text {ext }}\left|G^{(m)}\right|_{\text {ext }}
$$

Let $F^{(m)} \in \mathcal{H}_{\text {ext }}^{(m)}, f^{(n)} \in \mathcal{H}_{\text {ext }}^{(n)}, m>n$. We define a "product" $\left(f^{(n)}, F^{(m)}\right)_{\text {ext }} \in \mathcal{H}_{\text {ext }}^{(m-n)}$ by setting for each $g^{(m-n)} \in \mathcal{H}_{\text {ext }}^{(m-n)}$

$$
\left(g^{(m-n)},\left(f^{(n)}, F^{(m)}\right)_{\text {ext }}\right)_{\text {ext }}=\left(f^{(n)} \diamond g^{(m-n)}, F^{(m)}\right)_{\text {ext }} .
$$

Since by the Cauchy-Bunyakovsky inequality and (18)

$$
\left|\left(f^{(n)} \diamond g^{(m-n)}, F^{(m)}\right)_{\text {ext }}\right| \leq\left|f^{(n)} \diamond g^{(m-n)}\right|_{\text {ext }}\left|F^{(m)}\right|_{\text {ext }} \leq\left|f^{(n)}\right|_{\text {ext }}\left|g^{(m-n)}\right|_{\text {ext }}\left|F^{(m)}\right|_{\text {ext }},
$$

this definition is well-posed and

$$
\left|\left(f^{(n)}, F^{(m)}\right)_{\text {ext }}\right|_{\text {ext }} \leq\left|f^{(n)}\right|_{\text {ext }}\left|F^{(m)}\right|_{\text {ext }}
$$


Definition 6. Let $n \in \mathbb{N}, f^{(n)} \in \mathcal{H}_{\text {ext }}^{(n)}$. We define an operator of stochastic differentiation

$$
\left(D^{n} \circ\right)\left(f^{(n)}\right):\left(L^{2}\right)_{q}^{\beta} \rightarrow\left(L^{2}\right)_{q-1}^{\beta}
$$

by setting for $F \in\left(L^{2}\right)_{q}^{\beta}$

$$
\begin{aligned}
\left(D^{n} F\right)\left(f^{(n)}\right) & :=\sum_{m=n}^{\infty} \frac{m !}{(m-n) !}:\left\langle 0^{\otimes m-n},\left(f^{(n)}, F^{(m)}\right)_{\text {ext }}\right\rangle: \\
& \equiv \sum_{m=0}^{\infty} \frac{(m+n) !}{m !}:\left\langle 0^{\otimes m},\left(f^{(n)}, F^{(m+n)}\right)_{\text {ext }}\right\rangle:,
\end{aligned}
$$

where $F^{(m)} \in \mathcal{H}_{\text {ext }}^{(m)}$ are the kernels from decomposition (5) for $F$.

Using estimate (20) one can show [6] that this definition is well-posed and operator (21) is linear and continuous. Moreover, in the case $\beta=1$ formula (22) defines a linear continuous operator $\left(D^{n} \circ\right)\left(f^{(n)}\right)$ on $\left(L^{2}\right)_{q}^{1}, q \in \mathbb{Z}$.

Finally, as is easily seen, $\left(D^{n} \circ\right)\left(f^{(n)}\right)$ can be defined by formula (22) as a linear continuous operator on $\left(L^{2}\right)^{\beta}, \beta \in[-1,1]$. Namely a linear continuous operator

$$
(D \circ)(g):=\left(D^{1} \circ\right)(g):\left(L^{2}\right)^{-\beta} \rightarrow\left(L^{2}\right)^{-\beta}, \quad g \in \mathcal{H}_{\text {ext }}^{(1)}=\mathcal{H}_{\mathbb{C}}, \quad \beta \in[0,1],
$$

will be a subject of study in the forthcoming section.

Properties of operators of stochastic differentiation on spaces of regular test and generalized functions of the Lévy white noise analysis are considered in detail in $[6,7,9]$. Here we note only that the operator $D \equiv D^{1}$ and the Hida stochastic derivative are connected as follows [7]. Denote $\partial .:=1_{\mathbb{R}_{+}}(\cdot) \partial$.. Let $F \in\left(L^{2}\right)_{q}^{\beta}$ and $g \in \mathcal{H}_{\text {ext }}^{(1)}=\mathcal{H}_{\mathbb{C}}$. Then

$$
(D F)(g)=\int_{\mathbb{R}_{+}} \partial_{u} F \cdot g(u) d u \in\left(L^{2}\right)_{q-1^{\prime}}^{\beta}
$$

here the integral in the right hand side is a Pettis one (the weak integral). Taking into account this equality, one can write formally

$$
\partial . \circ=(D \circ)(\delta .)
$$

where $\delta$. is the Dirac delta-function concentrated at $\cdot$. In order to give a nonformal sense to equality (24), one can consider operators of stochastic differentiation on so-called spaces of nonregular generalized functions, see [18].

\section{ELEMENTS OF WicK CALCULUS}

\subsection{Wick product and Wick versions of holomorphic functions}

In this subsection we introduce and study a Wick product and Wick versions of holomorphic functions on $\left(L^{2}\right)^{-\beta}$, now $\beta \in[0,1]$.

First we give necessary definitions. 
Definition 7. For $F \in\left(L^{2}\right)^{-\beta}$ we define an $S$-transform $(S F)(\lambda), \lambda \in \mathcal{D}_{\mathbb{C}}$, as a formal series

$$
(S F)(\lambda):=\sum_{m=0}^{\infty}\left(F^{(m)}, \lambda^{\otimes m}\right)_{e x t} \equiv F^{(0)}+\sum_{m=1}^{\infty}\left(F^{(m)}, \lambda^{\otimes m}\right)_{e x t},
$$

where $F^{(m)} \in \mathcal{H}_{\text {ext }}^{(m)}, m \in \mathbb{Z}_{+}$, are the kernels from decomposition (5) for $F$ (each term in series (25) is well-defined, but the series can diverge). In particular, $(S F)(0)=F^{(0)}, S 1 \equiv 1$.

Definition 8. For $F, G \in\left(L^{2}\right)^{-\beta}$ and a holomorphic at $F^{(0)}$ function $h: \mathbb{C} \rightarrow \mathbb{C}$ we define a Wick product $F \diamond G$ and a Wick version $h^{\diamond}(F)$ by setting formally

$$
F \diamond G:=S^{-1}(S F \cdot S G), \quad h^{\diamond}(F):=S^{-1} h(S F) .
$$

Remark. It is obvious that the Wick product $\diamond$ is commutative, associative and distributive over a field $\mathbb{C}$.

A function $h$ from Definition 8 can be decomposed in a Taylor series

$$
h(u)=\sum_{m=0}^{\infty} h_{m}(u-(S F)(0))^{m} .
$$

Using this decomposition, it is easy to calculate that

$$
h^{\diamond}(F)=\sum_{m=0}^{\infty} h_{m}(F-(S F)(0))^{\diamond m},
$$

where $F^{\diamond m}:=\underbrace{F \diamond \cdots \diamond F}_{m \text { times }}, F^{\diamond 0}:=1$.

It easily follows from formula (2.23) in [19] that for $F^{(n)} \in \mathcal{H}_{\text {ext }}^{(n)}, G^{(m)} \in \mathcal{H}_{\text {ext }}^{(m)}, n, m \in \mathbb{Z}_{+}$, and $\lambda \in \mathcal{D}_{\mathbb{C}}$

$$
\left(F^{(n)}, \lambda^{\otimes n}\right)_{e x t}\left(G^{(m)}, \lambda^{\otimes m}\right)_{e x t}=\left(F^{(n)} \diamond G^{(m)}, \lambda^{\otimes n+m}\right)_{e x t}
$$

(a product $\diamond$ is defined in Subsection 1.4).

Using this formula, by analogy with the Meixner analysis [14] one can prove the following statement.

Proposition. For $F_{1}, \ldots, F_{n} \in\left(L^{2}\right)^{-\beta}$

$$
F_{1} \diamond \cdots \diamond F_{n}=\sum_{m=0}^{\infty}:\left\langle\diamond^{\otimes m}, \sum_{k_{1}, \ldots, k_{n} \in \mathbb{Z}_{+}: k_{1}+\cdots+k_{n}=m} F_{1}^{\left(k_{1}\right)} \diamond \cdots \diamond F_{n}^{\left(k_{n}\right)}\right\rangle:
$$

(in particular, for $F, G \in\left(L^{2}\right)^{-\beta} F \diamond G=\sum_{m=0}^{\infty}:\left\langle 0^{\otimes m}, \sum_{k=0}^{m} F^{(k)} \diamond G^{(m-k)}\right\rangle:$ ), where $F_{j}^{\left(k_{j}\right)} \in \mathcal{H}_{\text {ext }}^{\left(k_{j}\right)}$, $j \in\{1, \ldots, n\}, k_{j} \in \mathbb{Z}_{+}$, are the kernels from decompositions (5) for $F_{j} ; F^{(k)}, G^{(k)} \in \mathcal{H}_{\text {ext }}^{(k)}$, $k \in \mathbb{Z}_{+}$, are the kernels from the same decompositions for $F$ and $G$ respectively. Further, for $F \in\left(L^{2}\right)^{-\beta}$ and a holomorphic at $(S F)(0)=F^{(0)}$ function $h: \mathbb{C} \rightarrow \mathbb{C}$

$$
h^{\diamond}(F)=h_{0}+\sum_{m=1}^{\infty}:\left\langle\diamond^{\otimes m}, \sum_{n=1}^{m} h_{n} \sum_{k_{1}, \ldots, k_{n} \in \mathbb{N}: k_{1}+\cdots+k_{n}=m} F^{\left(k_{1}\right)} \diamond \cdots \diamond F^{\left(k_{n}\right)}\right\rangle:,
$$

where $F^{(k)} \in \mathcal{H}_{\text {ext }}^{(k)}, k \in \mathbb{Z}_{+}$, are the kernels from decomposition (5) for $F, h_{n} \in \mathbb{C}, n \in \mathbb{Z}_{+}$, are the coefficients from decomposition (27) for $h$. 
It is clear that in order to give a nonformal sense to notions "the Wick product" and "the Wick version of a holomorphic function", it is necessary to study a question about convergence of series (30) and (31) in the spaces of regular generalized functions. Using estimate (18), it is possible to do it as in the Meixner analysis [14]. As a result, for the Wick product we obtain the following statement (remind that now $\beta \in[0,1]$ ).

Theorem 1. Let $F_{1}, \ldots, F_{n} \in\left(L^{2}\right)^{-\beta}$. Then $F_{1} \diamond \cdots \diamond F_{n} \in\left(L^{2}\right)^{-\beta}$. Moreover, the Wick product is continuous in the topology of $\left(L^{2}\right)^{-\beta}$ : for arbitrary $F_{1}, \ldots, F_{n} \in\left(L^{2}\right)^{-\beta}, n \in \mathbb{N}$, there exist $q, q^{\prime} \in \mathbb{Z}_{+}\left(q>q^{\prime}+(1-\beta) \log _{2} n+1\right)$ such that

$$
\left\|F_{1} \diamond \cdots \diamond F_{n}\right\|_{-q,-\beta} \leq \sqrt{\max _{m \in \mathbb{Z}_{+}}\left[2^{-m}(m+1)^{n-1}\right]}\left\|F_{1}\right\|_{-q^{\prime},-\beta} \cdots\left\|F_{n}\right\|_{-q^{\prime},-\beta}
$$

(see (10)).

Now let us pass to consideration of the Wick versions of holomorphic functions. It follows from Theorem 1 and (28) that if $F \in\left(L^{2}\right)^{-\beta}$ and $h: \mathbb{C} \rightarrow \mathbb{C}$ is a polynomial then $h^{\diamond}(F) \in$ $\left(L^{2}\right)^{-\beta}$. But for a general $h$ the situation is more complicated: as in the Meixner analysis, the cases $\beta=1$ and $\beta \in[0,1)$ essentially differ. The case $\beta=1$ is comparatively simple: by analogy with [14] we obtain

Theorem 2. Let $F \in\left(L^{2}\right)^{-1}$ and a function $h: \mathbb{C} \rightarrow \mathbb{C}$ be holomorphic at $(S F)(0)$. Then $h^{\diamond}(F) \in\left(L^{2}\right)^{-1}$.

Let now $\beta \in[0,1)$. Since $\left(L^{2}\right)^{-\beta} \subset\left(L^{2}\right)^{-1}$, for $F \in\left(L^{2}\right)^{-\beta}$ and a holomorphic at $(S F)(0)$ function $h: \mathbb{C} \rightarrow \mathbb{C}$, by Theorem 2 the Wick version $h^{\diamond}(F)$ is a well-defined element of $\left(L^{2}\right)^{-1}$. But at the same time it is possible that $h^{\diamond}(F) \notin\left(L^{2}\right)^{-\beta}$, if $h$ is not a polynomial. More exactly, we have the following result.

Theorem 3. Let $u_{0} \in \mathbb{C}, h: \mathbb{C} \rightarrow \mathbb{C}$ be a holomorphic at $u_{0}$ function, which is not a polynomial and is such that all coefficients $h_{n}$ from the Taylor decomposition

$$
h(u)=\sum_{n=0}^{\infty} h_{n}\left(u-u_{0}\right)^{n}
$$

are real and non-negative. Then for each $\beta \in[0,1)$ one can find $F \in\left(L^{2}\right)^{-\beta}$ with $(S F)(0)=u_{0}$ such that $h^{\diamond}(F) \notin\left(L^{2}\right)^{-\beta}$.

The proof of this statement, in the same way as the proofs of Theorem 4 and Theorem 5 below, is completely analogous to the proof of the corresponding statement in the Meixner white noise analysis [14] and therefore can be omitted.

It follows from Theorem 3 that if $h$ is not a polynomial then, generally speaking, there are no estimates for coefficients from decomposition (32), which can guarantee that for arbitrary $F \in\left(L^{2}\right)^{-\beta}, \beta \in[0,1)$, with $(S F)(0)=u_{0}, h^{\diamond}(F)$ is an element of $\left(L^{2}\right)^{-\beta}$. Nevertheless, the following statement is valid.

Theorem 4. Let $F=\sum_{m=0}^{N}:\left\langle 0^{\otimes m}, F^{(m)}\right\rangle: \in\left(L^{2}\right)^{-\beta}, F^{(m)} \in \mathcal{H}_{\text {ext }}^{(m)}, N \in \mathbb{Z}_{+}$; and coefficients $h_{n} \in \mathbb{C}, n \in \mathbb{N}$, from the Taylor decomposition

$$
h(u)=\sum_{n=0}^{\infty} h_{n}\left(u-F^{(0)}\right)^{n}
$$


for a holomorphic at $F^{(0)} \in \mathbb{C}$ function $h: \mathbb{C} \rightarrow \mathbb{C}$ satisfy estimates

$$
\left|h_{n}\right| \leq \frac{K^{n}}{n^{n N \frac{1-\beta}{2}}}
$$

with some $K>0$. Then $h^{\diamond}(F) \in\left(L^{2}\right)^{-\beta}$.

Let now $0 \leq \beta_{1}<\beta_{2}<1$. We describe a sufficient condition under which $h^{\diamond}(F) \in\left(L^{2}\right)^{-\beta_{2}}$ for $F \in\left(L^{2}\right)^{-\beta_{1}}$ (note that if $\beta_{2}=1$ then by Theorem $2 h^{\diamond}(F) \in\left(L^{2}\right)^{-1}$ without additional conditions).

Theorem 5. Let $0 \leq \beta_{1}<\beta_{2}<1, F \in\left(L^{2}\right)^{-\beta_{1}}, h: \mathbb{C} \rightarrow \mathbb{C}$ be a holomorphic at $(S F)(0)$ function. If there exists $K>0$ such that for arbitrary $n \in \mathbb{N}$

$$
\left|h_{n}\right| \leq \frac{K^{n}}{\max _{m \in \mathbb{N}: m \geq n}\left(\frac{n^{m \frac{1-\beta_{2}}{2}}}{\left(\left[\frac{m}{n}\right] !\right)^{n} \frac{\beta_{2}-\beta_{1}}{2}}\right)}
$$

where $h_{n}$ are the coefficients from decomposition (27) for $h,[\cdot]$ denotes the integer part of a number, then $h^{\diamond}(F) \in\left(L^{2}\right)^{-\beta_{2}}$.

By analogy with the Meixner analysis [14] one can apply the above-formulated results for study of stochastic equations with Wick-type nonlinearities.

Example. Let us consider a stochastic equation

$$
X_{t}=X_{0}+\int_{0}^{t} X_{s} \diamond F d s+\int_{0}^{t} X_{s} \diamond G \widehat{d} L_{s}
$$

where $X_{0}, F, G \in\left(L^{2}\right)^{-\beta}, \int_{0}^{t} X_{s} \diamond F d s \in\left(L^{2}\right)^{-1}$ is a Pettis integral (the weak integral). Applying the $S$-transform and solving the obtained nonstochastic equation, we obtain

$$
S X_{t}=S X_{0} \cdot \exp \left\{S F t+S G \int_{0}^{t} \lambda(s) d s\right\}
$$

Now it is sufficient to apply the inverse S-transform in order to obtain the solution of (34)

$$
X_{t}=X_{0} \diamond \exp ^{\diamond}\left\{F t+G \diamond L_{t}\right\} \in\left(L^{2}\right)^{-1}
$$

(the fact that $X_{t} \in\left(L^{2}\right)^{-1}$ follows from Theorem 1 and Theorem 2). In order to obtain $X_{t} \in$ $\left(L^{2}\right)^{-\beta}, \beta<1$, we have to impose additional conditions. For example, let $F$ and $G$ be "polynomials" in the sense that their decompositions (5) contain only finite number of nonzero terms. Set $N:=\max [$ pow $F$, pow $G+1]$, where pow $H$ denotes the quantity of nonzero terms in decomposition (5) for $H$. If there exists $K>0$ such that for arbitrary $m \in \mathbb{N}$, where pow $H$ is the greatest number of nonzero term in decomposition,

$$
\frac{m^{m N \frac{1-\beta}{2}}}{m !} \leq K^{m},
$$

then by Theorem $4 X_{t} \in\left(L^{2}\right)^{-\beta}$ (see (33), now $h(u)=\exp (u), h_{m}=\frac{1}{m !}$ for each $m \in \mathbb{N}$ ). Note that estimates (35) are fulfilled if and only if $N \leq \frac{2}{1-\beta}$, this fact is proved in [14]. 


\subsection{Wick calculus and operators of stochastic differentiation}

In this subsection we consider an interconnection between the Wick calculus and the operator of stochastic differentiation $D$ (see (23)). In particular, we'll prove that this operator is a differentiation (satisfies the Leibniz rule) with respect to the Wick multiplication.

We define a characterization set of the space $\left(L^{2}\right)^{-\beta}$ in terms of the $S$-transform, setting $B_{\beta}:=S\left(L^{2}\right)^{-\beta} \equiv\left\{S F: F \in\left(L^{2}\right)^{-\beta}\right\}$. It is clear that $B_{\beta}$ is a linear space, which consists of formal series $\sum_{m=0}^{\infty}\left(F^{(m)}, \otimes^{\otimes m}\right)_{\text {ext }}$ (see (25)) with the kernels $F^{(m)} \in \mathcal{H}_{\text {ext }}^{(m)}$ satisfying a condition: there exists $q \in \mathbb{Z}_{+}$such that $\sum_{m=0}^{\infty}(m !)^{1-\beta} 2^{-q m}\left|F^{(m)}\right|_{\text {ext }}^{2}<\infty$. It follows from Definition 8 and Theorem 1 that $B_{\beta}$ is an algebra with respect to the pointwise multiplication.

Let $g \in \mathcal{H}_{\text {ext }}^{(1)}=\mathcal{H}_{\mathbb{C}}$. We define a "directional derivative" $D_{g}^{\diamond}: B_{\beta} \rightarrow B_{\beta}$ as follows. Set for $(S F)(\cdot)=\sum_{m=0}^{\infty}\left(F^{(m)}, \otimes m\right)_{\text {ext }} \in B_{\beta}\left(F \in\left(L^{2}\right)^{-\beta}, F^{(m)} \in \mathcal{H}_{\text {ext }}^{(m)}\right.$ are the kernels from decomposition (5) for $F$ )

$$
\left(D_{g}^{\diamond} S F\right)(\cdot):=\sum_{m=0}^{\infty}(m+1)\left(F^{(m+1)}, g \diamond\left(\cdot{ }^{\otimes m}\right)\right)_{\text {ext }}=\sum_{m=0}^{\infty}(m+1)\left(\left(g, F^{(m+1)}\right)_{\text {ext }}, \cdot{ }^{\otimes m}\right)_{\text {ext }} \in B_{\beta}
$$

(see (19)). Since $S^{-1}\left(D_{g}^{\diamond} S F\right)=\sum_{m=0}^{\infty}(m+1):\left\langle 0^{\otimes m},\left(g, F^{(m+1)}\right)_{\text {ext }}\right\rangle:=(D F)(g) \in\left(L^{2}\right)^{-\beta}$ (see (22)), the operator $D_{g}^{\diamond}$ is well-defined and the following statement is valid.

Proposition. The operator of stochastic differentiation $(D \circ)(g), g \in \mathcal{H}_{\text {ext }}^{(1)}=\mathcal{H}_{\mathrm{C}}$, is the preimage of the "directional derivative" $D_{g}^{\diamond}$ of $S \circ$ under the $S$-transform, i.e. for all $F \in\left(L^{2}\right)^{-\beta}$

$$
(D F)(g)=S^{-1}\left(D_{g}^{\diamond} S F\right) \in\left(L^{2}\right)^{-\beta} .
$$

Remark. If we introduce on $B_{\beta}$ a topology induced by the (inductive limit) topology of $\left(L^{2}\right)^{-\beta}$, then the $S$-transform will be a topological isomorphism between a topological algebra $\left(L^{2}\right)^{-\beta}$ with the Wick multiplication and a topological algebra $B_{\beta}$ with the pointwise multiplication. Now the "directional derivative" $D_{g}^{\diamond}, g \in \mathcal{H}_{\text {ext }}^{(1)}$, is the image on $B_{\beta}$ of the operator of stochastic differentiation $(D \circ)(g)$ on $\left(L^{2}\right)^{-\beta}$ (under the $S$-transform). Of course, $D_{g}^{\diamond}: B_{\beta} \rightarrow B_{\beta}$ is a linear continuous operator.

The main result of this subsection is the following

Theorem 6. The operator of stochastic differentiation $D$ is a differentiation with respect to the Wick multiplication, i.e. for arbitrary $F, G \in\left(L^{2}\right)^{-\beta}$ and $g \in \mathcal{H}_{\text {ext }}^{(1)}=\mathcal{H}_{\mathrm{C}}$

$$
(D(F \diamond G))(g)=(D F)(g) \diamond G+F \diamond(D G)(g) \in\left(L^{2}\right)^{-\beta} .
$$

Proof. First we note that the expressions in the left hand side and in the right hand side of (38) belong to $\left(L^{2}\right)^{-\beta}$, this follows from the definition of operator (23) and Theorem 1 . Let us prove the equality (38). By (37) and the first formula in (26)

$$
\begin{aligned}
& (D(F \diamond G))(g)=S^{-1}\left(D_{g}^{\diamond}(S(F \diamond G))\right)=S^{-1}\left(D_{g}^{\diamond}(S F \cdot S G)\right), \\
& (D F)(g) \diamond G=S^{-1}(S(D F)(g) \cdot S G)=S^{-1}\left(D_{g}^{\diamond}(S F) \cdot S G\right), \\
& F \diamond(D G)(g)=S^{-1}(S F \cdot S(D G)(g))=S^{-1}\left(S F \cdot D_{g}^{\diamond}(S G)\right),
\end{aligned}
$$


therefore it is sufficient to prove that

$$
D_{g}^{\diamond}(S F \cdot S G)=D_{g}^{\diamond}(S F) \cdot S G+S F \cdot D_{g}^{\diamond}(S G) .
$$

Let $F^{(m)}, G^{(m)} \in \mathcal{H}_{\text {ext }}^{(m)}$ be the kernels from decompositions (5) for $F$ and $G$ respectively. Using (25), (29) and (36), we obtain

$$
\begin{aligned}
& (S F)(\lambda)=\sum_{n=0}^{\infty}\left(F^{(n)}, \lambda^{\otimes n}\right)_{\text {ext }},(S G)(\lambda)=\sum_{m=0}^{\infty}\left(G^{(m)}, \lambda^{\otimes m}\right)_{\text {ext }}, \\
& (S F)(\lambda) \cdot(S G)(\lambda)=\sum_{n, m=0}^{\infty}\left(F^{(n)} \diamond G^{(m)}, \lambda^{\otimes n+m}\right)_{\text {ext }}, \\
& D_{g}^{\diamond}((S F)(\lambda) \cdot(S G)(\lambda))=\sum_{n, m=0}^{\infty}(n+m)\left(F^{(n)} \diamond G^{(m)}, g \diamond \lambda^{\otimes n+m-1}\right)_{\text {ext }}, \\
& D_{g}^{\diamond}(S F)(\lambda)=\sum_{n=0}^{\infty} n\left(F^{(n)}, g \diamond \lambda^{\otimes n-1}\right)_{\text {ext }}, D_{g}^{\diamond}(S G)(\lambda)=\sum_{m=0}^{\infty} m\left(G^{(m)}, g \diamond \lambda^{\otimes m-1}\right)_{\text {ext }}, \\
& D_{g}^{\diamond}(S F)(\lambda) \cdot(S G)(\lambda)=\sum_{n, m=0}^{\infty} n\left(F^{(n)}, g \diamond \lambda^{\otimes n-1}\right)_{\text {ext }}\left(G^{(m)}, \lambda^{\otimes m}\right)_{\text {ext }}, \\
& (S F)(\lambda) \cdot D_{g}^{\diamond}(S G)(\lambda)=\sum_{n, m=0}^{\infty} m\left(F^{(n)}, \lambda^{\otimes n}\right)_{\text {ext }}\left(G^{(m)}, g \diamond \lambda^{\otimes m-1}\right)_{\text {ext }},
\end{aligned}
$$

here $\lambda \in \mathcal{D}_{\mathrm{C}}$. So, in order to prove (39), it is sufficient to show that for all $n, m \in \mathbb{Z}_{+}$

$$
\begin{aligned}
(n+m)\left(F^{(n)} \diamond G^{(m)}, g \diamond \lambda^{\otimes n+m-1}\right)_{\text {ext }} & =n\left(F^{(n)}, g \diamond \lambda^{\otimes n-1}\right)_{\text {ext }}\left(G^{(m)}, \lambda^{\otimes m}\right)_{\text {ext }} \\
& +m\left(F^{(n)}, \lambda^{\otimes n}\right)_{\text {ext }}\left(G^{(m)}, g \diamond \lambda^{\otimes m-1}\right)_{\text {ext }} .
\end{aligned}
$$

It is easy to see that for $n=0$ or $m=0$ equality (40) is valid, therefore we consider the case $n, m \in \mathbb{N}$ only.

Let us consider $(n+m)\left(F^{(n)} \diamond G^{(m)}, g \diamond \lambda^{\otimes n+m-1}\right)_{\text {ext }}$. Denote by $\dot{f}^{(n)} \in F^{(n)}$ and $\dot{g}^{(m)} \in$ $G^{(m)}$ representatives of the equivalence classes $F^{(n)}$ and $G^{(m)}$. Set $\widehat{f^{(n)} g^{(m)}}:=\widetilde{f^{(n)} \cdot \dot{g}^{(m)}}$ (an operation $\widetilde{o}$ is defined in (17)). Let $\widehat{f^{(n) g^{(m)}}}$ be the symmetrization of $\widehat{f^{(n)} g^{(m)}}$ with respect to all arguments. We remind that $F^{(n)} \diamond G^{(m)}$ is an equivalence class in $\mathcal{H}_{\text {ext }}^{(n+m)}$ that is generated by $\widehat{f^{(n) g^{(m)}}}: \widehat{f^{(n) g^{(m)}}} \in F^{(n)} \diamond G^{(m)}$. Similarly for $\lambda \in \mathcal{D}_{\mathbb{C}}$ and a representative $\dot{g} \in g, g \in \mathcal{H}_{\text {ext }}^{(1)}$, set $\lambda^{\otimes n+m-1} g:=\lambda^{\otimes n+m-1} \cdot \dot{g}$, and denote by $\widehat{\lambda^{\otimes n+m-1}} \cdot g$ the symmetrization of $\lambda^{\otimes n+m-1} g$ with respect to all arguments. Then $\lambda^{\otimes n+m-1} g \in g \diamond \lambda^{\otimes n+m-1}$ (an equivalence class $g \diamond \lambda^{\otimes n+m-1} \in$ $\mathcal{H}_{\text {ext }}^{(n+m)}$ is generated by $\lambda^{\otimes n+m-1} g$ ).

Without loss of generality, one can assume that $\dot{f}^{(n)}$ and $\dot{g}^{(m)}$ are symmetric functions, and $m \geq n$. Taking this into consideration, we obtain

$$
\begin{aligned}
& \widehat{f^{(n) g^{(m)}}}\left(u_{1}, \ldots, u_{n} ; u_{n+1}, \ldots, u_{n+m}\right)=\frac{n ! m !}{(n+m) !} \\
& \times \sum_{\substack{1 \leq p_{1}, \ldots, p_{n} \leq n, n+1 \leq q_{1}, \ldots, q_{m} \leq n+m \\
0 \leq r \leq n, p_{1}<\cdots<p_{r}, p_{r+1}<\cdots<p_{n}, q_{1}<\cdots<q_{n-r}, q_{n-r+1}<\cdots<q_{m}}}^{f(n) g^{(m)}}\left(u_{p_{1}}, \ldots, u_{p_{r}}, u_{q_{1}}, \ldots, u_{q_{n-r}} ;\right. \\
& u_{\left.p_{r+1}, \ldots, u_{p_{n}}, u_{q_{n-r+1}}, \ldots, u_{q_{m}}\right),}
\end{aligned}
$$


here for $r=n$ the argument in the right hand side of $(41)$ is $\left(u_{1}, \ldots, u_{n} ; u_{n+1}, \ldots, u_{n+m}\right)$; for $r=$ 0 this argument is $\left(u_{q_{1}}, \ldots, u_{q_{n}} ; u_{1}, \ldots, u_{n}, u_{q_{n+1}}, \ldots, u_{q_{m}}\right)$ (see [7] for a detailed explanation).

Substituting (41) in the left hand side of (40), we obtain (see (6))

$$
\begin{aligned}
& (n+m)\left(F^{(n)} \diamond G^{(m)}, g \diamond \lambda^{\otimes n+m-1}\right)_{\text {ext }}=(n+m)\left(\widehat{f^{(n)} g^{(m)}}, \lambda^{\widehat{\otimes n+m-1}} g\right)_{\text {ext }} \\
& =(n+m) \sum_{\substack{k, l_{j}, s_{j} \in \mathbb{N}: j=1, \ldots, k_{,} l_{1}>l_{2}>\cdots>l_{k^{\prime}} \\
l_{1} s_{1}+\cdots+l_{k} s_{k}=n+m}} \frac{(n+m) !}{s_{1} ! \cdots s_{k} !}\left(\frac{\left\|p_{l_{1}}\right\|_{v}}{l_{1} !}\right)^{2 s_{1}} \cdots\left(\frac{\left\|p_{l_{k}}\right\|_{v}}{l_{k} !}\right)^{2 s_{k}} \\
& \times \int_{\mathbb{R}_{+}^{s_{1}+\cdots+s_{k}}} \widehat{f^{(n) g^{(m)}}}(\underbrace{u_{1}, \ldots, u_{1}}_{l_{1}}, \ldots, \underbrace{u_{s_{1}+\cdots+s_{k}} \ldots, u_{s_{1}+\cdots+s_{k}}}_{l_{k}}) \\
& \times\left(\lambda^{\widehat{\otimes n+m-1}} g\right)(\underbrace{u_{1}, \cdots, u_{1}}_{l_{1}}, \ldots, \underbrace{u_{s_{1}+\cdots+s_{k}}, \ldots, u_{s_{1}+\cdots+s_{k}}}_{l_{k}}) d u_{1} \cdots d u_{s_{1}+\cdots+s_{k}}= \\
& =(n+m) \sum_{\substack{k, l_{j}, s_{j} \in \mathbb{N}: j=1, \ldots, k_{,} l_{1}>l_{2}>\cdots>l_{k} \\
l_{1} s_{1}+\cdots+l_{k} s_{k}=n+m}} \frac{n ! m !}{s_{1} ! \cdots s_{k} !}\left(\frac{\left\|p_{l_{1}}\right\|_{v}}{l_{1} !}\right)^{2 s_{1}} \cdots\left(\frac{\left\|p_{l_{k}}\right\|_{v}}{l_{k} !}\right)^{2 s_{k}} \\
& \times\left[\int_{\mathbb{R}_{+}^{s_{1}+\cdots+s_{k}}} \widehat{f^{(n) g^{(m)}}}(\underbrace{u_{1}, \ldots, u_{1}}_{l_{1}}, \ldots, \underbrace{u_{s_{1}+\cdots+s_{k}}, \ldots, u_{s_{1}+\cdots+s_{k}}}_{l_{k}})\right. \\
& \left.\times\left(\lambda^{\otimes \widehat{n+m-1}} g\right)(\underbrace{u_{1}, \ldots, u_{1}}_{l_{1}}, \ldots, \underbrace{u_{s_{1}+\cdots+s_{k^{\prime}}} \ldots, u_{s_{1}+\cdots+s_{k}}}_{l_{k}}) d u_{1} \cdots d u_{s_{1}+\cdots+s_{k}}+\ldots\right] .
\end{aligned}
$$

We say that a collection of equal among one another arguments (e.g., $\left.\left(u_{1}, \ldots, u_{1}\right)\right)$ is called a procession. It follows from the ordering in ascending of indexes in (41) and in (6) that processions in summands in interior sums $[\cdots]$ from (42) can "tear" only so that different parts of a "torn" procession will be for different parties from ';;; processions being for one side from ';' do not switch places; and elements in processions do not switch places. In addition, it follows from a construction of $\widehat{f^{(n)} g^{(m)}}$ (see (17)) that summands in interior sums [...] from (42), in which a procession is divided by ' $;$ ', are equal to zero. Another summands (if there exist for a collection $k, l_{j}, s_{j}$ ) disintegrate on groups of equal among one another integrals. These groups arise by means of transpositions of processions with equal quantity of members, which are placed before ';' and after ' $;$ ', an equality of integrals under such transpositions from the symmetric property of a function $\lambda^{\otimes n+m-1} \mathrm{~g}$ follows: this symmetry gives a possibility to transpose mutually processions with equal quantity of members in the argument of $\widehat{\lambda^{\otimes n+m-1}} g$. It is clear that if there are $s^{\prime}$ processions of length $l$ before ';' and $s^{\prime \prime}$ processions of length $l$ after ';' tnen by means of mutual transpositions of these processions one can obtain $\frac{\left(s^{\prime}+s^{\prime \prime}\right) !}{s^{\prime} ! s^{\prime \prime} !}$ equal summands.

So, nonzero terms in the last expression in (42) are "connected" with equalities

$$
l_{1} s_{1}+\cdots+l_{k} s_{k}=n+m,
$$

that can be presented in the form

$$
\begin{gathered}
l_{1}^{\prime} s_{1}^{\prime}+\cdots+l_{k^{\prime}}^{\prime} s_{k^{\prime}}^{\prime}=n, l_{1}^{\prime \prime} s_{1}^{\prime \prime}+\cdots+l_{k^{\prime \prime}}^{\prime \prime} s_{k^{\prime \prime}}^{\prime \prime}=m, \\
k^{\prime}, k^{\prime \prime}, l_{1}^{\prime}, \ldots, l_{k^{\prime}}^{\prime} s_{1}^{\prime}, \ldots, s_{k^{\prime}}^{\prime}, l_{1}^{\prime \prime}, \ldots, l_{k^{\prime \prime}}^{\prime \prime}, s_{1}^{\prime \prime}, \ldots, s_{k^{\prime \prime}}^{\prime \prime} \in \mathbb{N}, \\
l_{1}^{\prime}>\cdots>l_{k^{\prime}}^{\prime}, l_{1}^{\prime \prime}>\cdots>l_{k^{\prime \prime}}^{\prime \prime}
\end{gathered}
$$


(the first sum in (44) corresponds to first $n$ arguments of $\widetilde{f^{(n)} g^{(m)}}$, the second sum corresponds to last $m$ arguments) as follows. For each $s_{j}$ from (43) either there exists $s_{i}^{\prime}=s_{j}\left(l_{i}^{\prime}=l_{j}\right)$ or there exists $s_{i}^{\prime \prime}=s_{j}\left(l_{i}^{\prime \prime}=l_{j}\right)$ or there exist $s_{i}^{\prime}$ and $s_{w}^{\prime \prime}$ such that $s_{i}^{\prime}+s_{w}^{\prime \prime}=s_{j}\left(l_{i}^{\prime}=l_{w}^{\prime \prime}=l_{j}\right)$. Inequalities for $l^{\prime}, l^{\prime \prime}$ in (44) follow from inequalities $l_{1}>\cdots>l_{k}$ and ordering of indexes in (41) and (6) (more long processions have smaller indexes of arguments).

We will replace each group of the above-described equal among one another integrals in the right hand side of (42) by a representative multiplied by a quantity of terms in the group. Also, since the Lebesgue measure is non-atomic, we can replace here $\widehat{f^{(n)} g^{(m)}}$ by $\dot{f}^{(n)} \cdot \dot{g}^{(m)}$ (in summands that remain elements of each procession are placed on the same side of ';'). Now, taking into account that $w^{s^{\prime}+s^{\prime \prime}}=w^{s^{\prime}} w^{s^{\prime \prime}}$, one can rewrite the last expression in (42) in the form

$$
\begin{aligned}
& \sum_{l_{1}^{\prime} s_{1}^{\prime}+\cdots+l_{k^{\prime}}^{\prime} s_{k^{\prime}}^{\prime}=n, l_{1}^{\prime \prime} s_{1}^{\prime \prime}+\cdots+l_{k^{\prime \prime}}^{\prime \prime} s_{k^{\prime \prime}}^{\prime \prime}=m,} \frac{n ! m !(n+m)}{s_{1}^{\prime} ! \cdots s_{k^{\prime}}^{\prime} s_{1}^{\prime \prime} ! \cdots s_{k^{\prime \prime}}^{\prime \prime} !}
\end{aligned}
$$

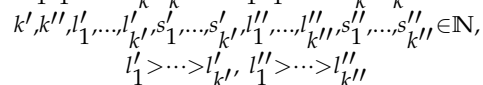

$$
\begin{aligned}
& \times\left(\frac{\left\|p_{l_{1}^{\prime}}\right\|_{v}}{l_{1}^{\prime} !}\right)^{2 s_{1}^{\prime}} \cdots\left(\frac{\left\|p_{l_{k^{\prime}}}\right\|_{v}}{l_{k^{\prime}}^{\prime} !}\right)^{2 s_{k^{\prime}}^{\prime}}\left(\frac{\left\|p_{l_{1}^{\prime \prime}}\right\|_{v}}{l_{1}^{\prime \prime} !}\right)^{2 s_{1}^{\prime \prime}} \cdots\left(\frac{\left\|p_{l_{k^{\prime \prime}}^{\prime \prime}}\right\|_{v}}{l_{k^{\prime \prime}}^{\prime \prime} !}\right)^{2 s_{k^{\prime \prime}}^{\prime \prime}} \\
& \times \int_{\mathbb{R}_{+} s_{1}^{\prime}+\cdots+s_{k^{\prime}}^{\prime}+s_{1}^{\prime \prime}+\cdots+s_{k^{\prime \prime}}^{\prime \prime}} \dot{f}^{(n)}(\underbrace{u_{1}, \ldots, u_{1}}_{l_{1}^{\prime}}, \ldots, \underbrace{u_{s_{1}^{\prime}+\cdots+s_{k^{\prime}}^{\prime}} \ldots, u_{s_{1}^{\prime}+\cdots+s_{k^{\prime}}^{\prime}}^{\prime}}_{l_{k^{\prime}}^{\prime}}) \\
& \times \dot{g}^{(m)}(\underbrace{u_{n+1}, \ldots, u_{n+1}}_{l_{1}^{\prime \prime}}, \cdots, \underbrace{\left.u_{n+s_{1}^{\prime \prime}+\cdots+s_{k^{\prime \prime}}^{\prime \prime}}, \ldots, u_{n+s_{1}^{\prime \prime}+\cdots+s_{k^{\prime \prime}}^{\prime \prime}}\right)}_{l_{k^{\prime \prime}}^{\prime \prime}} \\
& \times\left(\lambda^{\widehat{\otimes n+m-1}} g\right)(\underbrace{u_{1}, \ldots, u_{1}}_{l_{1}^{\prime}}, \ldots, \underbrace{u_{s_{1}^{\prime}+\cdots+s_{k^{\prime}}^{\prime}} \ldots, u_{s_{1}^{\prime}+\cdots+s_{k^{\prime}}^{\prime}}^{\prime}}_{l_{k^{\prime}}^{\prime}} \\
& \underbrace{u_{n+1}, \ldots, u_{n+1}}_{l_{1}^{\prime \prime}}, \cdots, \underbrace{\left.u_{n+s_{1}^{\prime \prime}+\cdots+s_{k^{\prime \prime}}^{\prime \prime}}, \ldots, u_{n+s_{1}^{\prime \prime}+\cdots+s_{k^{\prime \prime}}^{\prime \prime}}\right)}_{l_{k^{\prime \prime}}^{\prime \prime}} \\
& \times d u_{1} \cdots d u_{s_{1}^{\prime}+\cdots+s_{k^{\prime}}^{\prime}} d u_{n+1} \cdots d u_{n+s_{1}^{\prime \prime}+\cdots+s_{k^{\prime \prime}}^{\prime \prime}} \text {. }
\end{aligned}
$$

Further, the symmetrization of a function $\lambda^{\otimes n+m-1} \mathrm{~g}$ has a form

$$
\left(\lambda^{\widehat{\otimes n+m-1}} g\right)\left(u_{1}, \ldots, u_{n+m}\right)=\frac{1}{(n+m) !} \sum_{\pi \in S_{n+m}}\left(\lambda^{\otimes n+m-1} g\right)\left(u_{\pi(1)}, \ldots, u_{\pi(n+m)}\right)
$$

where $S_{n+m}$ is the set of all permutations of numbers $1, \ldots, n+m$. This representation can be essentially simplified if we take into account that $\widehat{\lambda^{\otimes n+m-1} g}$ is a symmetric function with respect to first $n+m-1$ arguments. Namely, consider all summands from (46) with the last argument $u_{n+m}$. It is clear that there are $(n+m-1)$ ! such summands, because they can be obtained by arbitrary permutations of arguments $u_{1}, \ldots, u_{n+m-1}$. Taking into account the abovementioned symmetry one can conclude that all these summands are equal among one another. So, it is possible to replace them by an arbitrary representative multiplied by $(n+m-1)$ !. Similarly one can group summands with the last arguments $u_{n+m-1}, u_{n+m-2}, \ldots, u_{1}$. Substituting 
multiplied by $(n+m-1)$ ! representatives of these groups of summands in (46), we obtain

$$
\begin{aligned}
& \left(\lambda^{\widehat{\otimes n+m-1}} g\right)\left(u_{1}, \ldots, u_{n+m}\right)=\frac{1}{(n+m)}\left[\left(\lambda^{\otimes n+m-1} g\right)\left(u_{1}, \ldots, u_{n+m}\right)\right. \\
& \left.+\left(\lambda^{\otimes n+m-1} g\right)\left(u_{n+m}, u_{1}, \ldots, u_{n+m-1}\right)+\ldots+\left(\lambda^{\otimes n+m-1} g\right)\left(u_{2}, \ldots, u_{n+m}, u_{1}\right)\right]
\end{aligned}
$$

(representatives of the above-described groups of summands are selected subject to consequent calculations).

Substituting (47) in (45), we obtain

$$
\begin{aligned}
& \sum_{l_{1}^{\prime} s_{1}^{\prime}+\cdots+l_{k^{\prime}}^{\prime} s_{k^{\prime}}^{\prime}=n, l_{1}^{\prime \prime} s_{1}^{\prime \prime}+\cdots+l_{k^{\prime \prime}}^{\prime \prime} s_{k^{\prime \prime}}^{\prime \prime}=m,} \frac{n ! m !}{s_{1}^{\prime} ! \cdots s_{k^{\prime}}^{\prime} ! s_{1}^{\prime \prime} ! \cdots s_{k^{\prime \prime}}^{\prime \prime} !}
\end{aligned}
$$

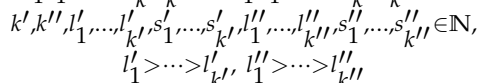

$$
\begin{aligned}
& \times\left(\frac{\left\|p_{l_{1}^{\prime}}\right\|_{v}}{l_{1}^{\prime} !}\right)^{2 s_{1}^{\prime}} \cdots\left(\frac{\left\|p_{l_{k^{\prime}}^{\prime}}\right\|_{v}}{l_{k^{\prime}}^{\prime} !}\right)^{2 s_{k^{\prime}}^{\prime}}\left(\frac{\left\|p_{l_{1}^{\prime \prime}}\right\|_{v}}{l_{1}^{\prime \prime} !}\right)^{2 s_{1}^{\prime \prime}} \cdots\left(\frac{\left\|p_{l_{k^{\prime \prime}}^{\prime \prime}}\right\|_{v}}{l_{k^{\prime \prime}}^{\prime \prime} !}\right)^{2 s_{k^{\prime \prime}}^{\prime \prime}}
\end{aligned}
$$

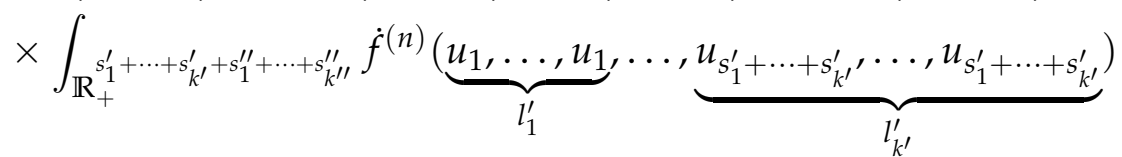

$$
\begin{aligned}
& \times \dot{g}^{(m)}(\underbrace{u_{n+1}, \ldots, u_{n+1}}_{l_{1}^{\prime \prime}}, \cdots, \underbrace{\left.u_{n+s_{1}^{\prime \prime}+\cdots+s_{k^{\prime \prime}}^{\prime \prime}}, \ldots, u_{n+s_{1}^{\prime \prime}+\cdots+s_{k^{\prime \prime}}^{\prime \prime}}\right)}_{l_{k^{\prime \prime}}^{\prime \prime}} \\
& \times\left[\left(\lambda^{\widehat{\otimes n+m-1}} g\right)(\underbrace{u_{1}, \ldots, u_{1}}_{l_{1}^{\prime}}, \ldots, \underbrace{u_{s_{1}^{\prime}+\cdots+s_{k^{\prime}}^{\prime}} \ldots, u_{s_{1}^{\prime}+\cdots+s_{k^{\prime}}^{\prime}}}_{l_{k^{\prime}}^{\prime}}\right.
\end{aligned}
$$

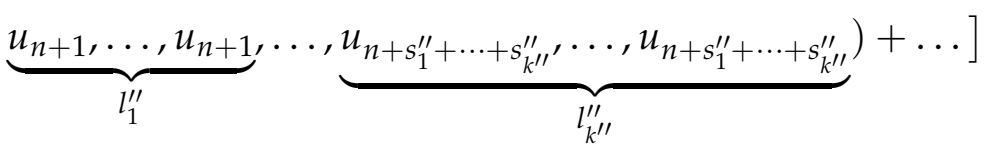

$$
\begin{aligned}
& \times d u_{1} \cdots d u_{s_{1}^{\prime}+\cdots+s_{k^{\prime}}^{\prime}} d u_{n+1} \cdots d u_{n+s_{1}^{\prime \prime}+\cdots+s_{k^{\prime \prime}}^{\prime \prime}}
\end{aligned}
$$

where each next term in the sum $[\cdots]$ with $n+m$ summands is obtained from the previous term by the "shift of arguments": $\left(\cdot 1, \ldots, \cdot n+m-1, \cdot_{n+m}\right) \rightarrow\left(\cdot_{n+m}, \cdot 1, \ldots, \cdot_{n+m-1}\right)$ etc. Taking into account the structure of $\lambda^{\otimes n+m-1} g$ (in particular, its symmetry with respect to first $n+$ $m-1$ arguments), the non-atomicity of the Lebesgue measure, and equalities (47) for $\lambda^{\otimes m-1} g$ and $\widehat{\lambda^{\otimes n-1}} g$, we can continue (48) as follows:

$$
\begin{aligned}
& (n+m)\left(F^{(n)} \diamond G^{(m)}, g \diamond \lambda^{\otimes n+m-1}\right)_{\text {ext }} \\
& =\sum_{l_{1}^{\prime} s_{1}^{\prime}+\cdots+l_{k^{\prime}}^{\prime} s_{k^{\prime}}^{\prime}=n, l_{1}^{\prime \prime} s_{1}^{\prime \prime}+\cdots+l_{k^{\prime \prime}}^{\prime \prime} s_{k^{\prime \prime}}^{\prime \prime}=m,} \frac{n ! m !}{s_{1}^{\prime} ! \cdots s_{k^{\prime}}^{\prime} ! s_{1}^{\prime \prime} ! \cdots s_{k^{\prime \prime}}^{\prime \prime} !} \\
& k^{\prime}, k^{\prime \prime}, l_{1}^{\prime}, \ldots, l_{k^{\prime}}^{\prime \prime}, s_{1}^{\prime}, \ldots, s_{k^{\prime}}^{\prime}, l_{1}^{\prime \prime}, \ldots, l_{k^{\prime \prime}}^{\prime \prime \prime}, s_{1}^{\prime \prime}, \ldots, s_{k^{\prime \prime}}^{\prime \prime} \in \mathbb{N}, \\
& \times\left(\frac{\left\|p_{l_{1}^{\prime}}\right\|_{v}}{l_{1}^{\prime} !}\right)^{2 s_{1}^{\prime}} \cdots\left(\frac{\left\|p_{l_{k^{\prime}}^{\prime}}\right\|_{v}}{l_{k^{\prime}}^{\prime} !}\right)^{2 s_{k^{\prime}}^{\prime}}\left(\frac{\left\|p_{l_{1}^{\prime \prime}}\right\|_{v}}{l_{1}^{\prime \prime} !}\right)^{2 s_{1}^{\prime \prime}} \cdots\left(\frac{\left\|p_{l_{k^{\prime \prime}}^{\prime \prime}}\right\|_{v}}{l_{k^{\prime \prime}}^{\prime \prime} !}\right)^{2 s_{k^{\prime \prime}}^{\prime \prime}}
\end{aligned}
$$

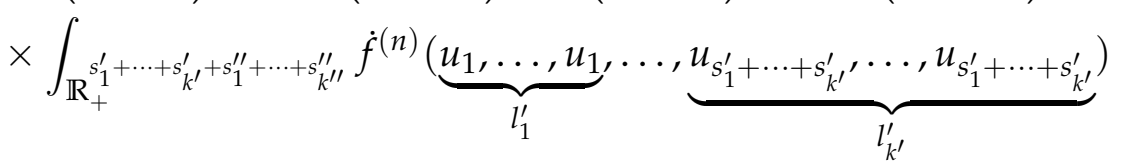




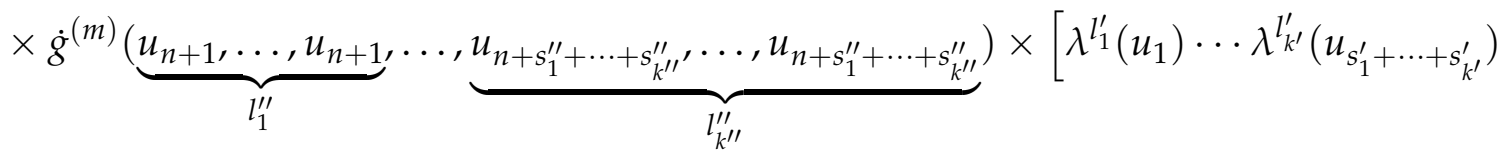

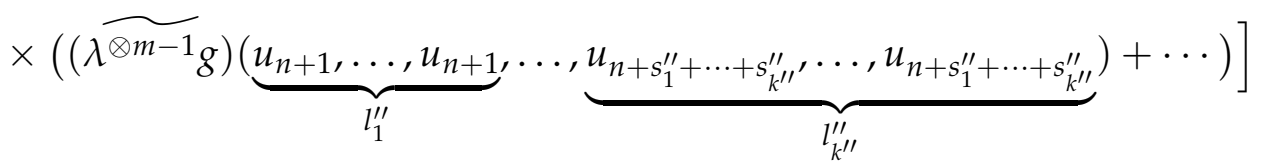$$
\times d u_{1} \cdots d u_{s_{1}^{\prime}+\cdots+s_{k^{\prime}}^{\prime}} d u_{n+1} \cdots d u_{n+s_{1}^{\prime \prime}+\cdots+s_{k^{\prime \prime}}^{\prime \prime}}
$$$$
+\sum_{l_{1}^{\prime} s_{1}^{\prime}+\cdots+l_{k^{\prime}}^{\prime} s_{k^{\prime}}^{\prime}=n, l_{1}^{\prime \prime} s_{1}^{\prime \prime}+\cdots+l_{k^{\prime \prime}}^{\prime \prime} s_{k^{\prime \prime}}^{\prime \prime}=m,} \frac{n ! m !}{s_{1}^{\prime} ! \cdots s_{k^{\prime}}^{\prime} ! s_{1}^{\prime \prime} ! \cdots s_{k^{\prime \prime}}^{\prime \prime} !}
$$

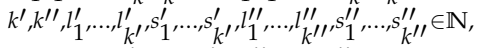$$
\times\left(\frac{\left\|p_{l_{1}^{\prime}}\right\|_{v}}{l_{1}^{\prime} !}\right)^{2 s_{1}^{\prime}} \cdots\left(\frac{\left\|p_{l_{k^{\prime}}^{\prime}}\right\|_{v}}{l_{k^{\prime}}^{\prime} !}\right)^{2 s_{k^{\prime}}^{\prime}}\left(\frac{\left\|p_{l_{1}^{\prime \prime}}\right\|_{v}}{l_{1}^{\prime \prime} !}\right)^{2 s_{1}^{\prime \prime}} \cdots\left(\frac{\left\|p_{l_{k^{\prime \prime}}^{\prime \prime}}\right\|_{v}}{l_{k^{\prime \prime}}^{\prime \prime} !}\right)^{2 s_{k^{\prime \prime}}^{\prime \prime}}
$$

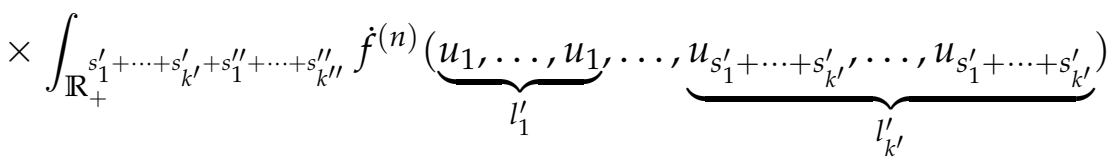$$
\times \dot{g}^{(m)}(\underbrace{u_{n+1}, \ldots, u_{n+1}}_{l_{1}^{\prime \prime}}, \cdots, \underbrace{\left.u_{n+s_{1}^{\prime \prime}+\cdots+s_{k^{\prime \prime}}^{\prime \prime}}, \ldots, u_{n+s_{1}^{\prime \prime}+\cdots+s_{k^{\prime \prime}}^{\prime \prime}}\right)}_{l_{k^{\prime \prime}}^{\prime \prime}}
$$$$
\times\left[\left(\left(\widehat{\lambda^{\otimes n-1} g}\right)(\underbrace{u_{1}, \ldots, u_{1}}_{l_{1}^{\prime}}, \ldots, \underbrace{u_{s_{1}^{\prime}+\cdots+s_{k^{\prime}}^{\prime}}, \ldots, u_{s_{1}^{\prime}+\cdots+s_{k^{\prime}}^{\prime}}}_{l_{k^{\prime}}^{\prime}})+\cdots\right)\right.
$$$$
\left.\times \lambda^{l_{1}^{\prime \prime}}\left(u_{n+1}\right) \cdots \lambda \lambda_{k^{\prime \prime}}^{l^{\prime \prime}}\left(u_{n+s_{1}^{\prime \prime}+\cdots+s_{k^{\prime \prime}}^{\prime \prime}}\right)\right] d u_{1} \cdots d u_{s_{1}^{\prime}+\cdots+s_{k^{\prime}}^{\prime}} d u_{n+1} \cdots d u_{n+s_{1}^{\prime \prime}+\cdots+s_{k^{\prime \prime}}^{\prime \prime}}
$$$$
=m\left(\dot{f}^{(n)}, \lambda^{\otimes n}\right)_{\text {ext }}\left(\dot{g}^{(m)}, \widehat{\lambda}^{\otimes m-1} g\right)_{\text {ext }}+n\left(\dot{f}^{(n)}, \widehat{\lambda^{\otimes n-1}} g\right)_{\text {ext }}\left(\dot{g}^{(m)}, \lambda^{\otimes m}\right)_{\text {ext }}
$$$$
=m\left(F^{(n)}, \lambda^{\otimes n}\right)_{\text {ext }}\left(G^{(m)}, g \diamond \lambda^{\otimes m-1}\right)_{\text {ext }}+n\left(F^{(n)}, g \diamond \lambda^{\otimes n-1}\right)_{\text {ext }}\left(G^{(m)}, \lambda^{\otimes m}\right)_{\text {ext }} .
$$

So, (40) is fulfilled, hence (39) is valid and therefore equality (38) is proved.

Corollary. Let $F \in\left(L^{2}\right)^{-\beta}, g \in \mathcal{H}_{\text {ext }}^{(1)}=\mathcal{H}_{\mathbb{C}}$, and $h: \mathbb{C} \rightarrow \mathbb{C}$ be a holomorphic at $(S F)(0)$ function. Then

$$
\left(D h^{\diamond}(F)\right)(g)=h^{\prime \diamond}(F) \diamond(D F)(g) \in\left(L^{2}\right)^{-1},
$$

where $h^{\prime \diamond}$ is the Wick version of the usual derivative of a function $h$.

Proof. First we'll prove by the mathematical induction method that for each $m \in \mathbb{Z}_{+}$

$$
\left(D(F-(S F)(0))^{\diamond m}\right)(g)=m(F-(S F)(0))^{\diamond m-1} \diamond(D F)(g) .
$$

In fact, in the case $m=0$ equality (50) is, obviously, true (we remind that $(F-(S F)(0))^{\diamond 0}=1$ by definition and for $G \in \mathbb{C} \subset\left(L^{2}\right)^{-\beta} D G=0$ ). Let us suppose that (50) is valid for $m \leq k$, $k \in \mathbb{Z}_{+}$. In particular,

$$
\left(D(F-(S F)(0))^{\diamond k}\right)(g)=k(F-(S F)(0))^{\diamond k-1} \diamond(D F)(g) .
$$

We have to show that

$$
\left(D(F-(S F)(0))^{\diamond k+1}\right)(g)=(k+1)(F-(S F)(0))^{\diamond k} \diamond(D F)(g) .
$$


Using (38) and (51) we obtain

$$
\begin{aligned}
& \left(D(F-(S F)(0))^{\diamond k+1}\right)(g)=\left(D\left[(F-(S F)(0))^{\diamond k} \diamond(F-(S F)(0))\right]\right)(g) \\
& =\left(D(F-(S F)(0))^{\diamond k}\right)(g) \diamond(F-(S F)(0))+(F-(S F)(0))^{\diamond k} \diamond(D(F-(S F)(0)))(g) \\
& =k(F-(S F)(0))^{\diamond k-1} \diamond(D F)(g) \diamond(F-(S F)(0))+(F-(S F)(0))^{\diamond k} \diamond(D F)(g) \\
& =k(F-(S F)(0))^{\diamond k} \diamond(D F)(g)+(F-(S F)(0))^{\diamond k} \diamond(D F)(g) \\
& =(k+1)(F-(S F)(0))^{\diamond k} \diamond(D F)(g),
\end{aligned}
$$

which is what had to be proved.

Further, consider decomposition (28) for $h^{\diamond}(F)$. Let $h_{N}^{\diamond}(F):=\sum_{m=0}^{N} h_{m}(F-(S F)(0))^{\diamond m}$ be the $N$-th partial sum of this decomposition. It follows from the linearity of $D,(50)$, and Theorems 2 and 1 that

$$
\begin{aligned}
\left(D h_{N}^{\diamond}(F)\right)(g) & =\sum_{m=1}^{N} h_{m}\left(D(F-(S F)(0))^{\diamond m}\right)(g) \\
& =\sum_{m=1}^{N} h_{m} m(F-(S F)(0))^{\diamond m-1} \diamond(D F)(g) \underset{N \rightarrow \infty}{\rightarrow} h^{\prime \diamond}(F) \diamond(D F)(g)
\end{aligned}
$$

in $\left(L^{2}\right)^{-1}$, where $h^{\prime \diamond}$ is the Wick version of the usual derivative of a function $h$. On the other hand, since $(D \circ)(g)$ is a continuous operator on $\left(L^{2}\right)^{-1},\left(D h_{N}^{\diamond}(F)\right)(g) \underset{N \rightarrow \infty}{\rightarrow}\left(D h^{\diamond}(F)\right)(g)$ in $\left(L^{2}\right)^{-1}$. So, equality (49) is valid.

\section{REFERENCES}

[1] Benth F.E., Di Nunno G., Lokka A., Oksendal B., Proske F. Explicit representation of the minimal variance portfolio in markets driven by Lévy processes. Math. Finance 2003, 13 (1), 55-72. doi: 10.1111/1467-9965.t01-1-00005

[2] Berezansky Yu.M., Kondratiev Yu.G. Spectral methods in infinite-dimensional analysis. In: Mathematical physics and applied mathematics, 12 (1-2). Kluwer Academic Publishers, Dordrecht, 1995.

[3] Berezansky Yu. M., Sheftel Z.G., Us G.F. Functional analysis. In: Ball J.A., Böttcher A., Dym H. (Eds.) Operator theory: advances and applications, 85 (1). Birkhäuser Verlag, Basel, 1996.

[4] Di Nunno G., Oksendal B., Proske F. Malliavin calculus for Lévy processes with applications to finance. In: Axler S., Casacuberta C., MacIntyre A. Universitext. Springer-Verlag, Berlin, 2009.

[5] Di Nunno G., Oksendal B., Proske F. White noise analysis for Léry processes. J. Funct. Anal. 2004, 206 (1), 109 148. doi: 10.1016/S0022-1236(03)00184-8

[6] Dyriv M.M., Kachanovsky N.A. On operators of stochastic differentiation on spaces of regular test and generalized functions of Lévy white noise analysis. Carpathian Math. Publ. 2014, 6 (2), 212-229. doi: 10.15330/cmp.6.2.212229

[7] Dyriv M.M., Kachanovsky N.A. Operators of stochastic differentiation on spaces of regular test and generalized functions in the Léry white noise analysis. KPI Sci. News 2014, 4, 36-40.

[8] Dyriv M.M., Kachanovsky N.A. Stochastic integrals with respect to a Levy process and stochastic derivatives on spaces of regular test and generalized functions. KPI Sci. News 2013, 4, 27-30.

[9] Frei M.M., Kachanovsky N.A. Some remarks on operators of stochastic differentiation in the Léry white noise analysis. Methods Funct. Anal. Topology 2017, 23 (4), 320-345. 
[10] Hida T. Analysis of Brownian Functionals. In: Carleton mathematical lecture notes, Vol. 13. Carleton University, Ottava, 1975.

[11] Holden H., Oksendal B., Uboe J., Zhang T. Stochastic partial differential equations: a modeling, white noise functional approach. Birkhäuser, Boston, 1996.

[12] Itô K. Spectral type of the shift transformation of differential processes with stationary increments. Trans. Amer. Math. Soc. 1956, 81 (1), 253-263. doi: 10.1090/S0002-9947-1956-0077017-0

[13] Kabanov Yu.M., Skorohod A.V. Extended stochastic integrals. In: Proc. School-Seminar "Theory of Random Procesess", Druskininkai, Lietuvos Respublika, November 25-30, 1974, Inst. Phys. Math., Vilnius, 1975, 123167. (in Russian)

[14] Kachanovsky N.A. An extended stochastic integral and a Wick calculus on parametrized Kondratiev-type spaces of Meixner white noise. Infin. Dimens. Anal. Quantum Probab. Relat. Top. 2008, 11 (4), 541-564. doi: 10.1142/S0219025708003270

[15] Kachanovsky N.A. Extended stochastic integrals and Wick calculus on spaces of regular generalized functions connected with Gamma measure. Ukrainian Math. J. 2005, 57 (8), 1214-1248. doi: 10.1007/s11253-005-0258-8

[16] Kachanovsky N.A. Extended stochastic integrals with respect to a Lévy process on spaces of generalized functions. Math. Bull. Shevchenko Sci. Soc. 2013, 10, 169-188.

[17] Kachanovsky N.A. On extended stochastic integrals with respect to Lévy processes. Carpathian Math. Publ. 2013, 5 (2), 256-278. doi: 10.15330/cmp.5.2.256-278

[18] Kachanovsky N.A. Operators of stochastic differentiation on spaces of nonregular generalized functions of Lévy white noise analysis. Carpathian Math. Publ. 2016, 8 (1), 83-106. doi: 10.15330/cmp.8.1.83-106

[19] Kachanovsky N.A. Operators of stochastic differentiation on spaces of nonregular test functions of Léry white noise analysis. Methods Funct. Anal. Topology 2015, 21 (4), 336-360.

[20] Kachanovsky N.A., Tesko V.A. Stochastic integral of Hitsuda-Skorokhod type on the extended Fock space. Ukrainian Math. J. 2009, 61 (6), 873-907. doi: 10.1007/s11253-009-0257-2

[21] Kondratiev Yu.G. Generalized functions in problems of infinite-dimensional analysis. Ph. D. Thesis. Kyiv, 1978. (in Russian)

[22] Kondratiev Yu.G., Leukert P., Streit L. Wick calculus in Gaussian analysis. Acta Appl. Math. 1996, 44 (3), 269 294. doi: $10.1007 / \mathrm{BF} 00047395$

[23] Lytvynov E. Orthogonal decompositions for Lévy processes with an application to the gamma, Pascal, and Meixner processes. Infin. Dimens. Anal. Quantum Probab. Relat. Top. 2003, 6 (1), 73-102. doi: $10.1142 /$ S0219025703001031

[24] Nualart D., Schoutens W. Chaotic and predictable representations for Léry processes. Stochastic Process. Appl. 2000, 90 (1), 109-122. doi: 10.1016/S0304-4149(00)00035-1

[25] Schoutens W. Stochastic Processes and Orthogonal Polynomials. In: Bickel P., Diggle P. (Eds.) Lecture notes in statistics, 146 (1). Springer-Verlag, New York, 2000.

[26] Skorohod A.V. Integration in Hilbert Space. Springer-Verlag, New York and Heidelberg, 1974. (translation of Skorohod A.V. Integration in Hilbert Space. Nauka, Moskow, 1974.(in Russian))

[27] Skorohod A.V. On a generalization of a stochastic integral. Theory Probab. Appl. 1976, 20 (2), 219-233. (translation of Teor. Veroyatn. Primen. 1975, 20 (2), 223-238. (in Russian))

[28] Solé J.L., Utzet F., Vives J. Chaos expansions and Malliavin calculus for Lévy processes. In: Benth F.E., Di Nunno G. (Eds.) Stochastic analysis and applications. Abel symposia, 2. Springer, Heidelberg, $2007,595-612$. doi: 10.1007/978-3-540-70847-6_27

[29] Surgailis D. On $L^{2}$ and non- $L^{2}$ multiple stochastic integration. In: Arató M., Vermes D. Stochastic differential systems. Lecture notes in control and information sciences, 36. Springer-Verlag., Heidelberg, 1981, $212-226$. doi: $10.1007 / \mathrm{BFb} 0006424$ 
[30] Vershik A.M., Tsilevich N.V. Fock factorizations and decompositions of the $L^{2}$ spaces over general Lévy processes. Russian Math. Surveys 2003, 58 (3), 427-472. doi: 10.1070/RM2003v058n03ABEH000627

Received 26.11.2017

Revised 22.06.2018

Фрей М.М. Віківське числення на просторах регулярних узагальнених функцій аналізу білого шуму Иеві // Карпатські матем. публ. - 2018. - Т.10, №1. - С. 82-104.

Багато об'єктів Гауссівського аналізу білого шуму (простори основних і узагальнених функцій, стохастичні інтеграли та похідні, тощо) можна будувати і досліджувати у термінах так званих хаотичних розкладів, що базуються на властивості хаотичного розкладу (ВХР): грубо кажучи, кожну квадратично інтегровну відносно гауссівської міри випадкову величину можна розкласти у ряд стохастичних інтегралів Іто від невипадкових функцій. У аналізі Аеві нема BХР (крім гауссівського та пуассонівського частинних випадків). Тим не менш, існують різні узагальнення цієі властивості. Використовуючи ці узагальнення, можна будувати різні простори основних і узагальнених функцій. I у кожному випаАку необхідно уводити природний добуток на просторах узагальнених функцій, та вивчати пов'язані питання. Цей добуток називається віківсъким добутком, як у гауссівському аналізі.

Конструкція віківського добутку у аналізі $е$ еві залежить, зокрема, від обраного узагальнення ВХР. У цій статті ми маємо справу з литвинівським узагальненням ВХР та з відповідними просторами регулярних узагальнених функцій. Метою статті є увести та вивчити віківський добуток на цих просторах, та розглянути деякі пов'язані питання (віківські версії голоморфних функцій, взаємозв' язок віківського числення з операторами стохастичного диференціювання). Основні результати статті полягають у вивченні властивостей віківського добутку та віківських версій голоморфних функцій. Зокрема, ми довели, шо оператор стохастичного диференціювання є диференціюванням (задовольняє правило Аейбніца) відносно віківського множення.

Ключові слова і фрази: Процес Аеві, стохастичне диференціювання, віківський добуток. 